\title{
Equilibrium Points and Related Periodic Motions in the Restricted Three-Body Problem with Angular Velocity and Radiation Effects
}

\author{
E. A. Perdios, ${ }^{1}$ V. S. Kalantonis, ${ }^{1}$ A. E. Perdiou, ${ }^{2}$ and A. A. Nikaki ${ }^{3}$ \\ ${ }^{1}$ Department of Electrical \& Computer Engineering, University of Patras, 26500 Patras, Greece \\ ${ }^{2}$ Department of Civil Engineering, University of Patras, 26500 Patras, Greece \\ ${ }^{3}$ Department of Computer Engineering \& Informatics, University of Patras, 26500 Patras, Greece \\ Correspondence should be addressed to V. S. Kalantonis; kalantonis@upatras.gr
}

Received 24 April 2015; Accepted 2 June 2015

Academic Editor: Zdzislaw E. Musielak

Copyright (C) 2015 E. A. Perdios et al. This is an open access article distributed under the Creative Commons Attribution License, which permits unrestricted use, distribution, and reproduction in any medium, provided the original work is properly cited.

\begin{abstract}
The paper deals with a modification of the restricted three-body problem in which the angular velocity variation is considered in the case where the primaries are sources of radiation. In particular, the existence and stability of its equilibrium points in the plane of motion of the primaries are studied. We find that this problem admits the well-known five planar equilibria of the classical problem with the difference that the corresponding collinear points may be stable depending on the parameters of the problem. For all planar equilibria, sufficient parametric conditions for their stability have been established which are used for the numerical determination of the stability regions in various parametric planes. Also, for certain values of the parameters of the problem for which the equilibrium points are stable, the short and long period families have been computed. To do so, semianalytical expressions have been found for the determination of appropriate initial conditions. Special attention has been given to the continuation of the long period family, in the case of the classical restricted three-body problem, where we show numerically that periodic orbits of the short period family, which are bifurcation points with the long period family, are connected through the characteristic curve of the long period family.
\end{abstract}

\section{Introduction}

The restricted three-body problem is the most celebrated problem of Celestial Mechanics (see, among others, Szebehely [1], Marsden and Ross [2], and references therein) and due to its interdisciplinary applications it has also attracted the interest of many researchers from different fields of science (see, e.g., Prosmiti et al. [3], Vrahatis et al. [4], Sano [5]). During the last century, several modifications of this classical problem have been introduced in order to make it more relevant and applicable to certain systems of Dynamical Astronomy. In our present study, we will consider the combination of two such modifications in which the variation of the angular velocity (the Chermnykh's problem) and the radiation effects of the primaries (the photogravitational problem) are considered.

The Chermnykh's problem is a generalization of the Euler's problem of two fixed gravitational centers and the restricted three-body problem, in which the third body of negligible mass moves in the orbital plane of a dumbbell which is rotating with constant angular velocity $\omega$ around the center of mass (Chermnykh [6]). This problem admits applications in both Dynamical Astronomy and Celestial Mechanics as well as in Chemistry (see Goździewski and Maciejewski [7] and references therein) and has been studied recently by several authors. In particular, Goździewski and Maciejewski [7] investigated the nonlinear Lyapunov stability of the triangular equilibrium points. Perdios and Ragos [8] studied asymptotic motions around the collinear equilibrium points while Papadakis [9] considered planar and threedimensional periodic motions around them. Perdiou et al. [10] studied three-dimensional motions through bifurcations of the Sitnikov family. In recent years, certain variants of this problem have been also proposed by other authors. Specifically, Jiang and Yeh [11] investigated the equilibrium points of 
the Chermnykh's problem in the case where the value of the angular velocity is determined by the gravitational potential of the belt which was considered to be a part of the potential function. For the same variant Abouelmagd et al. [12] studied the linear stability of the triangular equilibrium points as well as periodic solutions around them when the primaries are radiating oblate spheroids. Recently, Singh and Leke $[13,14]$ considered also the oblateness of the test particle as well as the effect of small perturbations in the Coriolis and centrifugal forces.

On the other hand, an interesting modification of the classical problem is the photogravitational restricted three-body problem in which the repulsive force of the radiation is also considered in the potential function and it was introduced for studying the specific three-body problem of Sun, planet, and a dust particle (Radzievskii [15]). This is a simplified model, since it does not take into account the perturbation force of the Poynting-Robertson effect but only the radiation pressure as a reduction factor of the magnitude of the inverse square law forces of the primaries upon the massless body. However, it may be useful for the study of motion of extremely small particles such as dust grains and interplanetary drifters (see, e.g., Kalvouridis [16], Bewick et al. [17], and references therein). In the framework of this simplified model many works have been done in the last years. For example, Simmons et al. [18] studied the existence and stability of the equilibrium points for all values of the radiation factors and the mass parameter, Goździewski et al. [19] considered the nonlinear stability of the triangular equilibrium points, Elipe and Lara [20] studied three-dimensional periodic orbits, and Perdios et al. [21] investigated the bifurcations of plane to threedimensional periodic orbits, while Perdios [22] determined series of horizontally critical symmetric periodic orbits as well as their vertical stability. Also, Zimovshikov and Tkhai [23] studied the stability of the collinear equilibrium points in the elliptic case of the corresponding problem, Abouelmagd [24] considers the additional effects of the oblateness as well as the Coriolis and centrifugal forces on the location and stability of the equilibrium points, while Singh and Taura [25] investigated the linear stability of the triangular equilibrium points by taking into account the oblateness of the smaller primary and the gravitational potential from a circumbinary belt. Recently, Yárnoz et al. [26] examined the feasibility of manipulating asteroid material by means of solar radiation pressure, while Verrier et al. [27] studied the evolution of the $L_{1}$ halo family under the effects of radiation pressure in the Sun-Earth system.

In this paper, the equilibrium points as well as the periodic orbits around them are studied in the framework of the photogravitational Chermnykh's restricted three-body problem with the aim to investigate the changes which may result in these basic dynamical features due to the three parameters of this model-problem (the angular velocity $\omega$ and the radiation factors $q_{1}$ and $q_{2}$ of the primaries). In particular, we study the existence and location of the planar equilibrium points as well as their linear stability. An interesting result is that there are combinations of the parameters of the problem for which the collinear equilibrium points may be stable. This is established by certain conditions which are determined when the triangular equilibrium points fall on the $\mathrm{O} x$-axis and transfer their stability to the collinear points. Several stability regions are given to show this result. For all cases of stable collinear and triangular equilibrium points we compute the corresponding short and long period families. We also give emphasis on the computation of the long period family emanating from each equilateral equilibrium point, in the framework of the classical restricted three-body problem for the Sun-Jupiter system, showing numerically that the long period family constitutes a bridge connecting orbits of the short period family which are bifurcation points of these two families complementing thusly the results of Henrard [28].

Specifically, the paper is organized as follows. In Section 2, we introduce the equations of motion of the considered model-problem. In Section 3, the existence, location, and stability of planar equilibrium points are investigated while, in Section 4, periodic motions around stable collinear and triangular equilibrium points are studied. Finally, in Section 5, we summarize our results and conclude.

\section{Equations of Motion}

We consider a barycentric, rotating, and dimensionless coordinate system $\mathrm{O} x y$ whose origin is at the center of mass of two bodies $m_{1}=1-\mu$ and $m_{2}=\mu$, known as primaries, where $\mu=m_{2} /\left(m_{1}+m_{2}\right) \leq 1 / 2$ is the mass parameter. The two bodies perform circular orbits around the center of mass with angular velocity $\omega \geq 0$ and are always on the $\mathrm{O} x$-axis (Figure 1). They are also considered to be radiating sources with mass reduction factors due to the radiation $0<$ $q_{i} \leq 1, i=1,2$, respectively (Chernikov [29]). Thus, the motion of the third body of negligible mass is influenced by the gravitational force as well as the repulsive force of light pressure. This additional force of radiation has applications in stellar dynamics, where it is a main component of the influence of a star on dust particles (Kunitsyn and Tureshbaev [30] and Das et al. [31]). Note that radiation factors may also have negative values meaning that gravity is strengthened by radiation, a case which will not be considered in our study. The equations of motion of the massless body are given by the following system (Schuerman [32], Chermnykh [6], and Perdios and Ragos [8]):

$$
\begin{aligned}
& \ddot{x}-2 \omega \dot{y}=x\left(\omega^{2}-Q^{*}\right)-\mu(1-\mu) R^{*}, \\
& \ddot{y}+2 \omega \dot{x}=y\left(\omega^{2}-Q^{*}\right),
\end{aligned}
$$

where we have abbreviated

$$
\begin{aligned}
& Q^{*}=\frac{q_{1}(1-\mu)}{r_{1}^{3}}+\frac{q_{2} \mu}{r_{2}^{3}}, \\
& R^{*}=\frac{q_{1}}{r_{1}^{3}}-\frac{q_{2}}{r_{2}^{3}},
\end{aligned}
$$




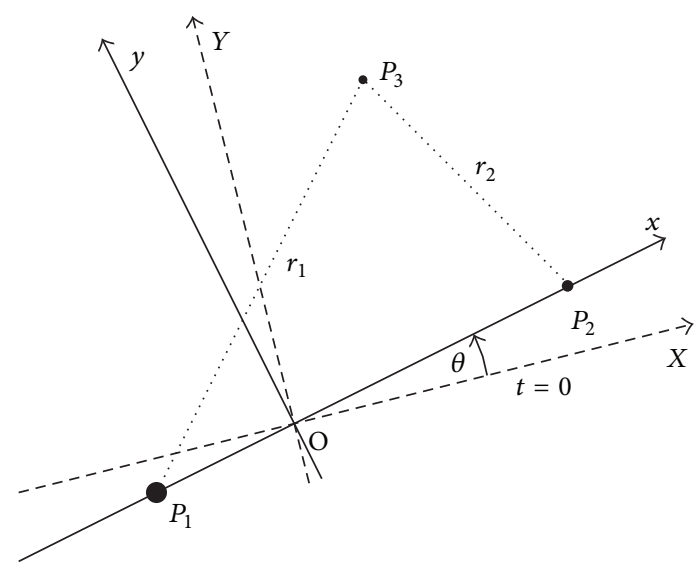

Figure 1: The rotating coordinate system $(\mathrm{O} x y)$ with angular velocity $\omega$ relative to the inertial frame $(\mathrm{OXY})$. The two primaries and the massless body are denoted by $P_{1,2}$ and $P_{3}$, respectively.

while $\Omega$ is the potential function which is given by the following formula:

$$
\begin{aligned}
& \Omega=\frac{\omega^{2}}{2}\left(x^{2}+y^{2}\right)+\frac{q_{1}(1-\mu)}{r_{1}}+\frac{q_{2} \mu}{r_{2}}, \\
& r_{1}^{2}=(x+\mu)^{2}+y^{2}, \\
& r_{2}^{2}=(x+\mu-1)^{2}+y^{2},
\end{aligned}
$$

are the distances of the third body of infinitesimal mass from the primary and secondary body, respectively. The system admits the Jacobi integral:

$$
C=2 \Omega-\left(\dot{x}^{2}+\dot{y}^{2}\right),
$$

where $C$ is the Jacobi constant.

\section{Equilibrium Points and Their Stability}

3.1. Collinear Equilibria. The Eulerian equilibrium points lie on the $\mathrm{O} x$-axis and may be determined from system (1) by setting the components of velocity and acceleration equal to zero as well as $y=0$ and by solving numerically for $x$ the nonlinear equation:

$$
\begin{aligned}
f(x) & =x\left(\omega^{2}-Q^{*}\right)-\mu(1-\mu) R^{*} \\
& =\omega^{2} x-\frac{(1-\mu) q_{1}(x+\mu)}{|x+\mu|^{3}}-\frac{\mu q_{2}(x+\mu-1)}{|x+\mu-1|^{3}} \\
& =0,
\end{aligned}
$$

for certain values of the four parameters of the problem, $q_{1}$, $q_{2}, \omega$, and $\mu$. The case where $\omega=1$, that is, the photogravitational version of the restricted three-body problem, has been studied extensively by Simmons et al. [18], where, for $0<q_{i}<1, i=1,2$, they show the existence of three collinear equilibrium points. The addition of the parameter of the angular velocity $\omega$ does not affect the total number of collinear equilibria and following the usual terminology existing in the literature, we name them as $L_{i}, i=1,2,3$. Their positions satisfy

$$
x_{L_{3}}<-\mu<x_{L_{1}}<1-\mu<x_{L_{2}}
$$

where $-\mu$ and $1-\mu$ are the locations of the larger and smaller primary, respectively. It is worth mentioning here the positions of these points, in the limiting case where the angular velocity parameter tends to infinity. So, supposing that $x \neq-\mu, 1-\mu$, and by elimination of the denominators in (6), we obtain that

$$
\begin{aligned}
0 & =\lim _{\omega \rightarrow \infty} \frac{1}{\omega^{2}} f(x) \\
& =x(x+\mu)(x+\mu-1)|x+\mu||x+\mu-1|,
\end{aligned}
$$

which means that the only acceptable solution is $x=0$; that is, only the inner equilibrium point still exists and it is located at the origin, while the outer collinear points approach the primaries as the value of the angular velocity parameter increases and disappears when $\omega \rightarrow \infty$.

3.2. Triangular Equilibria. For zero velocity and acceleration components in the equations of motion (1) we obtain

$$
\begin{array}{r}
x\left(\omega^{2}-Q^{*}\right)-\mu(1-\mu) R^{*}=0, \\
y\left(\omega^{2}-Q^{*}\right)=0,
\end{array}
$$

and by solving this system for $x$ and $y$, when $y \neq 0$, we are able to determine the positions of the Lagrangian equilibrium points. System (9) yields the following expressions:

$$
\begin{aligned}
& r_{1}=\left(\frac{q_{1}}{\omega^{2}}\right)^{1 / 3}, \\
& r_{2}=\left(\frac{q_{2}}{\omega^{2}}\right)^{1 / 3},
\end{aligned}
$$

and by substituting them into (4) we obtain, after some simple calculations, the exact positions of the triangular equilibrium points:

$$
\begin{aligned}
& x=\frac{1}{2}\left[1+\left(\frac{q_{1}}{\omega^{2}}\right)^{2 / 3}-\left(\frac{q_{2}}{\omega^{2}}\right)^{2 / 3}\right]-\mu, \\
& y= \pm\left\{\left(\frac{q_{1}}{\omega^{2}}\right)^{2 / 3}-\frac{1}{4}\left[1+\left(\frac{q_{1}}{\omega^{2}}\right)^{2 / 3}-\left(\frac{q_{2}}{\omega^{2}}\right)^{2 / 3}\right]^{2}\right\}^{1 / 2}
\end{aligned}
$$

It ensues from (10) that since $r_{1,2}>0$, these points exist for $q_{1,2}>0$ as well as for

$$
\begin{aligned}
r_{1}+r_{2}=\left(\frac{q_{1}}{\omega^{2}}\right)^{1 / 3}+\left(\frac{q_{2}}{\omega^{2}}\right)^{1 / 3} & \geq 1 \\
& \text { namely } Q_{1}+Q_{2} \geq \omega^{2 / 3},
\end{aligned}
$$


or

$$
\begin{aligned}
\left|r_{1}-r_{2}\right|=\left|\left(\frac{q_{1}}{\omega^{2}}\right)^{1 / 3}-\left(\frac{q_{2}}{\omega^{2}}\right)^{1 / 3}\right| & \leq 1 \\
& \text { namely }\left|Q_{1}-Q_{2}\right| \leq \omega^{2 / 3},
\end{aligned}
$$

where we set $Q_{1}=q_{1}^{1 / 3}$ and $Q_{2}=q_{2}^{1 / 3}$. The (limit) equality in (12), that is, $Q_{1}+Q_{2}=\omega^{2 / 3}$, shows that the triangular equilibria have coalesced with the inner collinear equilibrium point $L_{1}$ while the equalities in (13), that is, $Q_{1}-Q_{2}=\omega^{2 / 3}$ or $Q_{2}-Q_{1}=\omega^{2 / 3}$, indicate that the triangular points have coalesced with the outer collinear equilibrium points $L_{2}$ or $L_{3}$, respectively. Summarizing, the necessary conditions for the existence of planar equilibrium points, with $y \neq 0$, which simultaneously have to be satisfied are

$$
\begin{gathered}
Q_{1}>0, \\
Q_{2}>0, \\
\left|Q_{1}-Q_{2}\right|<\omega^{2 / 3}<Q_{1}+Q_{2} .
\end{gathered}
$$

We mention here that in the limiting case where the angular velocity parameter tends to infinity these points do not exist as this may be directly seen from (10). Note also that for $\omega=1$ inequality (12) is in agreement with Schuerman [32] while (13) cannot be established in the photogravitational version since the triangular equilibria do not fall on any outer collinear point.

3.3. Stability of Equilibria. In order to study their stability we transfer the origin at an equilibrium point $L_{i}, i=1,2, \ldots, 5$ :

$$
\begin{aligned}
& x=x_{0}+\xi, \\
& y=y_{0}+\eta,
\end{aligned}
$$

and we expand the equations of motion (1) into first-order terms with respect to $\xi$ and $\eta$ obtaining the linearized system:

$$
\begin{aligned}
& \dot{\Xi}=A \Xi, \\
& \Xi=(\xi, \eta, \dot{\xi}, \dot{\eta})^{\mathrm{T}},
\end{aligned}
$$

where the matrix of variations is

$$
A=\left[\begin{array}{cccc}
0 & 0 & 1 & 0 \\
0 & 0 & 0 & 1 \\
\Omega_{x x}^{0} & \Omega_{x y}^{0} & 0 & 2 \omega \\
\Omega_{y x}^{0} & \Omega_{y y}^{0} & -2 \omega & 0
\end{array}\right],
$$

with

$$
\begin{aligned}
& \Omega_{x x}^{0}=\omega^{2}-Q_{0}^{*}-x_{0} Q_{1}^{*}-\mu(1-\mu) R_{1}^{*}, \\
& \Omega_{y y}^{0}=\omega^{2}-Q_{0}^{*}-y_{0} Q_{2}^{*}, \\
& \Omega_{x y}^{0}=-x_{0} Q_{2}^{*}-\mu(1-\mu) R_{2}^{*}, \\
& \Omega_{y x}^{0}=-y_{0} Q_{1}^{*},
\end{aligned}
$$

where we have abbreviated

$$
\begin{aligned}
& Q_{1}^{*}=-\frac{3 q_{1}(1-\mu)\left(x_{0}+\mu\right)}{r_{10}^{5}}-\frac{3 q_{2} \mu\left(x_{0}+\mu-1\right)}{r_{20}^{5}}, \\
& Q_{2}^{*}=-\frac{3 q_{1}(1-\mu) y_{0}}{r_{10}^{5}}-\frac{3 q_{2} \mu y_{0}}{r_{20}^{5}}, \\
& R_{1}^{*}=-\frac{3 q_{1}\left(x_{0}+\mu\right)}{r_{10}^{5}}+\frac{3 q_{2}\left(x_{0}+\mu-1\right)}{r_{20}^{5}}, \\
& R_{2}^{*}=-\frac{3 q_{1} y_{0}}{r_{10}^{5}}+\frac{3 q_{2} y_{0}}{r_{20}^{5}}
\end{aligned}
$$

while $Q_{0}^{*}, R_{0}^{*}$ are given by (2) and $r_{10}, r_{20}$ by (4) evaluated at the equilibrium point $\left(x_{0}, y_{0}\right)$. The characteristic equation is quadratic with respect to $\Lambda=\lambda^{2}$ and is given by

$$
\begin{aligned}
\Lambda^{2} & +\left(4 \omega^{2}-\Omega_{x x}^{0}-\Omega_{y y}^{0}\right) \Lambda+\left(\Omega_{x x}^{0} \Omega_{y y}^{0}-\Omega_{x y}^{0} \Omega_{y x}^{0}\right) \\
& =0
\end{aligned}
$$

For the collinear equilibrium points $\Omega_{x y}^{0}=\Omega_{y x}^{0}=0$, and stability occurs when all roots of the characteristic equation for $\lambda$ are imaginary. Therefore, for stability the following three necessary and sufficient conditions must be fulfilled simultaneously:

$$
\begin{aligned}
4 \omega^{2}-\Omega_{x x}^{0}-\Omega_{y y}^{0} & >0, \\
\Omega_{x x}^{0} \Omega_{y y}^{0} & >0, \\
8 \omega^{2}\left(2 \omega^{2}-\Omega_{x x}^{0}-\Omega_{y y}^{0}\right)+\left(\Omega_{x x}^{0}-\Omega_{y y}^{0}\right)^{2} & >0,
\end{aligned}
$$

which ensure that the characteristic equation (20) has two negative real roots $\Lambda_{1,2}$; that is, the characteristic equation for $\lambda$ has four pure imaginary roots. The characteristic equation (20) may be written in the following form:

$$
\Lambda^{2}+\left(2 \omega^{2}-Q_{0}^{*}\right) \Lambda+\left[\omega^{4}+\omega^{2} Q_{0}^{*}-2\left(Q_{0}^{*}\right)^{2}\right]=0,
$$

and inequalities (21) lead to the double inequality for $Q_{0}^{*}$ :

$$
\frac{8 \omega^{2}}{9}<Q_{0}^{*}<\omega^{2}
$$

Also, by solving (6) for $Q_{0}^{*}$ we get for any collinear equilibrium point that

$$
Q_{0}^{*}=\frac{1}{\left(x_{0}+\mu\right)}\left(\omega^{2} x_{0}+\frac{\mu q_{2}}{\left|x_{0}+\mu-1\right|^{3}}\right) .
$$

Let us consider now the case of the outer collinear equilibrium point $L_{2}$ located in the open interval $(1-\mu,+\infty)$; that is, $\left|x_{0}+\mu-1\right|^{3}=\left(x_{0}+\mu-1\right)^{3}$. From (24) and the right inequality of (23) we easily obtain that

$$
q_{2}<\omega^{2}\left(x_{0}+\mu-1\right)^{3},
$$


and using this inequality we have from (6) that

$$
q_{1}>\omega^{2}\left(x_{0}+\mu\right)^{3} \text {. }
$$

Following the same procedure, we obtain from (24) and the left inequality of (23) and (6) that

$$
\begin{aligned}
& q_{2}>-\frac{\omega^{2}}{9 \mu}\left(x_{0}-8 \mu\right)\left(x_{0}+\mu-1\right)^{3}, \\
& q_{1}<\frac{\omega^{2}}{9(1-\mu)}\left(x_{0}-8 \mu+8\right)\left(x_{0}+\mu\right)^{3} .
\end{aligned}
$$

Therefore, in order to have stability for the collinear equilibrium point $L_{2}$ the following inequalities have to be satisfied:

$$
\begin{aligned}
& \omega^{2 / 3}\left(x_{0}+\mu\right)<Q_{1} \\
& <\frac{\omega^{2 / 3}}{[9(1-\mu)]^{1 / 3}}\left(x_{0}-8 \mu+8\right)^{1 / 3}\left(x_{0}+\mu\right), \\
& -\frac{\omega^{2 / 3}}{(9 \mu)^{1 / 3}}\left(x_{0}-8 \mu\right)^{1 / 3}\left(x_{0}+\mu-1\right)<Q_{2} \\
& <\omega^{2 / 3}\left(x_{0}+\mu-1\right),
\end{aligned}
$$

where we recall that we have set $Q_{1}=q_{1}^{1 / 3}$ and $Q_{2}=q_{2}^{1 / 3}$. Using a similar analysis for the outer collinear equilibrium point $L_{3}$ located in the interval $(-\infty,-\mu)$ we obtain that for stability the corresponding inequalities take the following form:

$$
\begin{aligned}
& -\frac{\omega^{2 / 3}}{[9(1-\mu)]^{1 / 3}}\left(x_{0}-8 \mu+8\right)^{1 / 3}\left(x_{0}+\mu\right)<Q_{1} \\
& <-\omega^{2 / 3}\left(x_{0}+\mu\right) \\
& -\omega^{2 / 3}\left(x_{0}+\mu-1\right)<Q_{2} \\
& <\frac{\omega^{2 / 3}}{(9 \mu)^{1 / 3}}\left(x_{0}-8 \mu\right)^{1 / 3}\left(x_{0}+\mu-1\right),
\end{aligned}
$$

while for the inner collinear equilibrium point $L_{1}$ located in the interval $(-\mu, 1-\mu)$ we get them in the following form:

$$
\begin{aligned}
& \frac{\omega^{2 / 3}}{[9(1-\mu)]^{1 / 3}}\left(x_{0}-8 \mu+8\right)^{1 / 3}\left(x_{0}+\mu\right)<Q_{1} \\
& <\omega^{2 / 3}\left(x_{0}+\mu\right), \\
& \frac{\omega^{2 / 3}}{(9 \mu)^{1 / 3}}\left(x_{0}-8 \mu\right)^{1 / 3}\left(x_{0}+\mu-1\right)<Q_{2} \\
& <-\omega^{2 / 3}\left(x_{0}+\mu-1\right) .
\end{aligned}
$$

To study the stability of the triangular equilibrium points it suffices to consider one of them, for example, the equilibrium point $L_{4}$, the position of which is given by (11) for the positive sign of the $y$ coordinate. So, by substituting its exact position into the characteristic equation (20) we obtain

$$
\begin{aligned}
& \Lambda^{2}+\omega^{2} \Lambda-\frac{9 \mu(1-\mu) \omega^{4}}{4 Q_{1}^{2} Q_{2}^{2}}\left[Q_{1}^{4}+\left(Q_{2}^{2}-\omega^{4 / 3}\right)^{2}\right. \\
& \left.-2 Q_{1}^{2}\left(Q_{2}^{2}+\omega^{4 / 3}\right)\right]=0 .
\end{aligned}
$$

Note here that, in the case of the triangular equilibrium points, the quantities $\Omega_{x y}^{0}=\Omega_{y x}^{0}$ in (20) are not zero. Therefore, in order for $L_{4}$ to be stable, inequalities (21) take now the following form:

$$
\begin{aligned}
& 2 Q_{1}^{2}\left(Q_{2}^{2}+\omega^{4 / 3}\right)-Q_{1}^{4}-\left(Q_{2}^{2}-\omega^{4 / 3}\right)^{2}>0, \\
& Q_{1}^{2} Q_{2}^{2}+9 \mu(1-\mu) \\
& \quad \cdot\left[Q_{1}^{4}+\left(Q_{2}^{2}-\omega^{4 / 3}\right)^{2}-2 Q_{1}^{2}\left(Q_{2}^{2}+\omega^{4 / 3}\right)\right]>0,
\end{aligned}
$$

which depend only on the four parameters of the problem. The latter of the above two inequalities arises from the discriminant of the characteristic equation (31) and may be written as a second degree polynomial with respect to $\mu$ :

$$
\alpha \mu^{2}+\beta \mu+\gamma
$$

where

$$
\begin{aligned}
& \alpha=-9\left[Q_{1}^{4}+\left(Q_{2}^{2}-\omega^{4 / 3}\right)^{2}-2 Q_{1}^{2}\left(Q_{2}^{2}+\omega^{4 / 3}\right)\right], \\
& \beta=-\alpha, \\
& \gamma=Q_{1}^{2} Q_{2}^{2} .
\end{aligned}
$$

When the discriminant is equal to zero, the characteristic equation has a double negative root which means that the triangular points are unstable. Thus, the roots of (33) provide the necessary condition for the mass parameter when stability alternates to instability and vice versa.

All the above analysis has been used to determine the stability regions of all equilibrium points of the model under consideration. So, in Figure 2 we present the stability regions in the $\left(Q_{1}, Q_{2}\right)$ plane for $\omega=0.25$ and for six different values of the mass parameter. Stability of the collinear equilibrium points is shown with light grey color, while dark grey color indicates stability of the triangular equilibrium points and white color corresponds to instability. Note here that the straight continuous lines correspond to the equalities of (12) and (13); that is, $\left|Q_{1} \pm Q_{2}\right|=\omega^{2 / 3}$, indicating that the triangular equilibrium points have coalesced to a collinear equilibrium point. So, we can see from this figure that the unstable, in the case of the classical restricted three-body problem, collinear equilibrium points become stable when the stable triangular equilibrium points fall on the $x$-axis. This means that the stability of the triangular equilibrium points is transferred to the collinear equilibrium points when $\left|Q_{1} \pm Q_{2}\right|=\omega^{2 / 3}$ and is lost from the dashed curves of the figure representing the rest of the inequalities of (28), (29), and (30). In particular, in the first frame of this figure which 


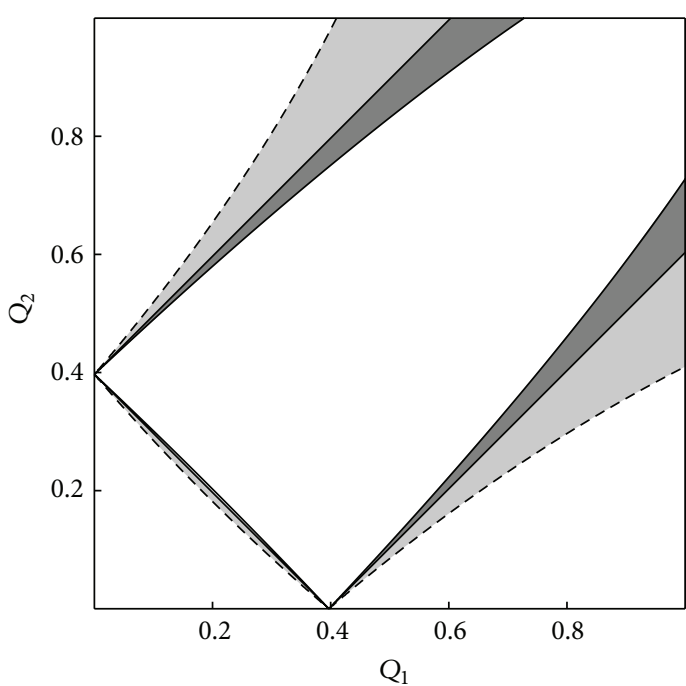

(a)

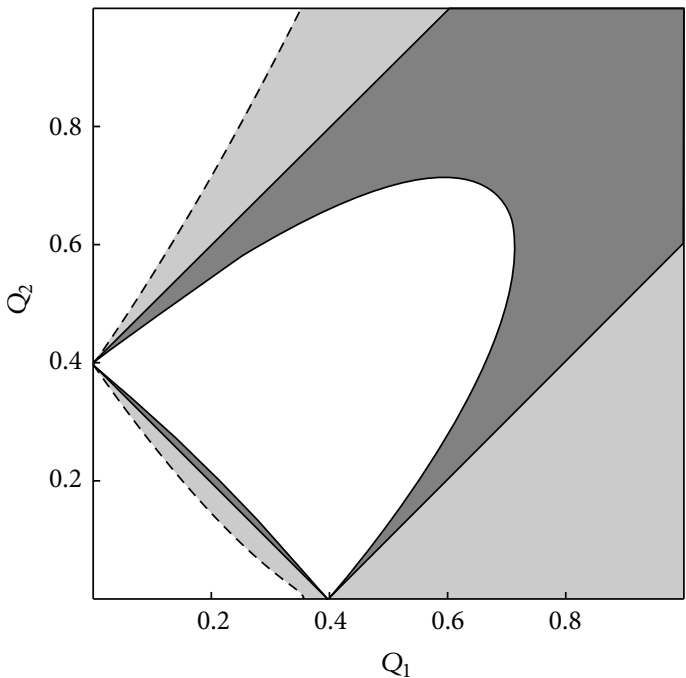

(c)

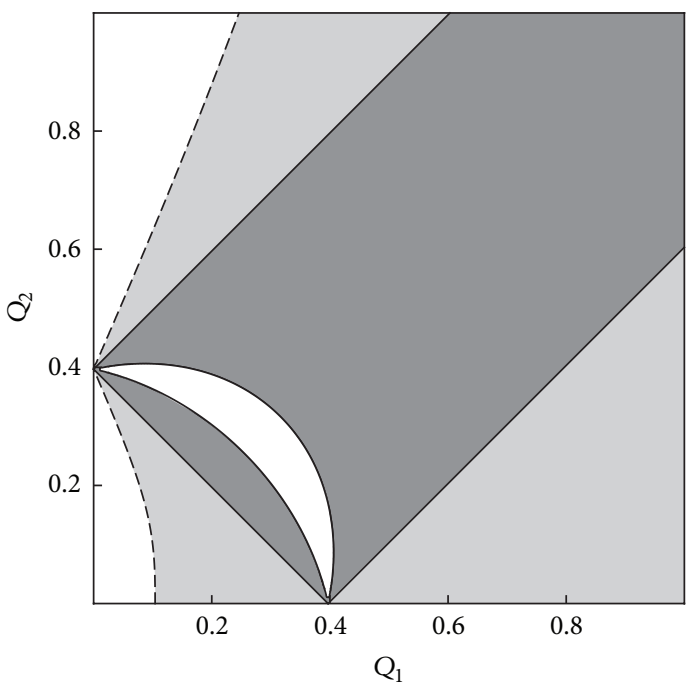

(e)

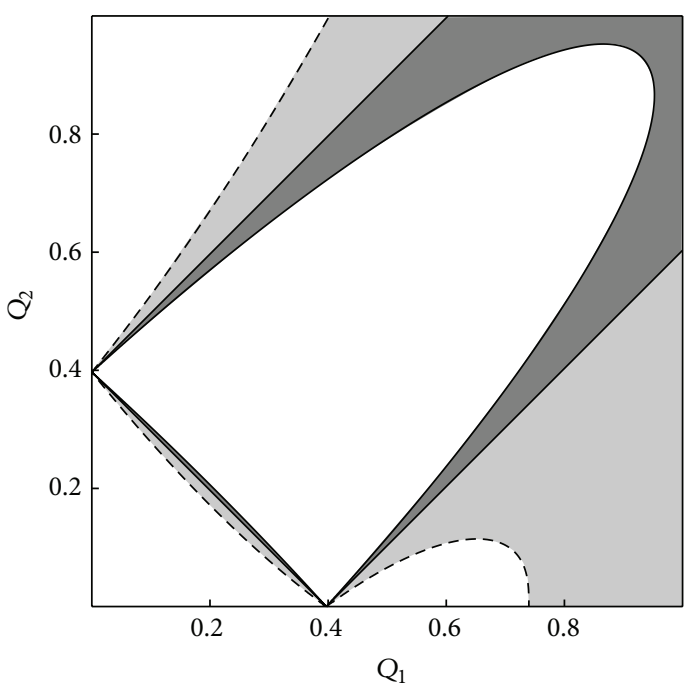

(b)

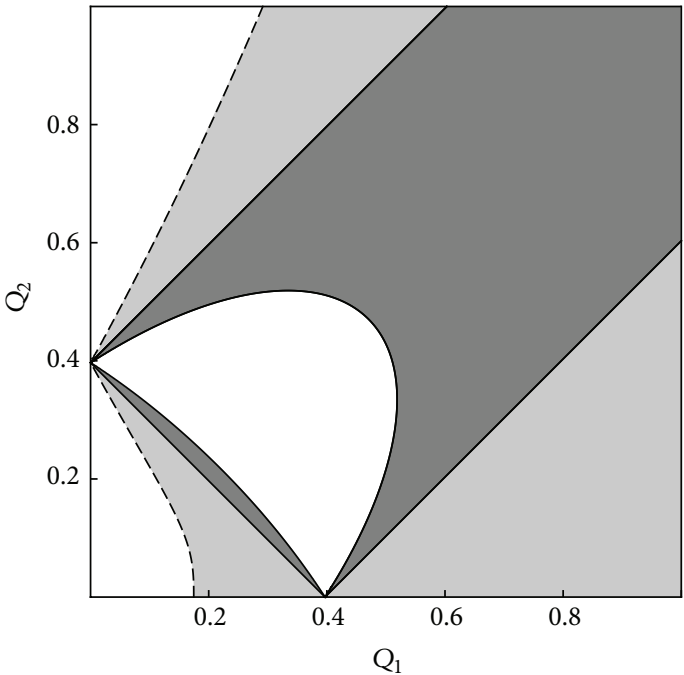

(d)

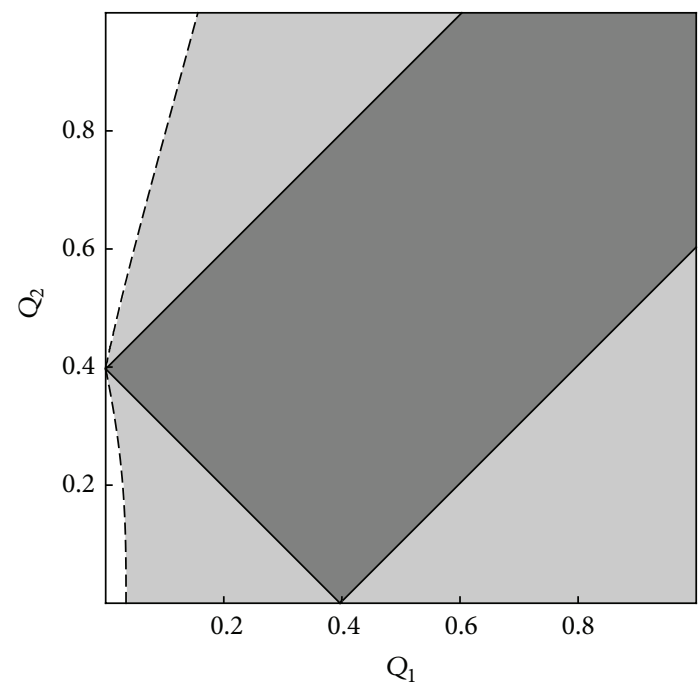

(f)

Figure 2: Stability regions of all equilibrium points in the $\left(Q_{1}, Q_{2}\right)$ plane for $\omega=0.25$ and $\mu=0.5, \mu=0.2, \mu=0.1$, (from (a) to (c)) $\mu=0.05, \mu=0.03$, and $\mu=0.01$ (from (d) to (f)). 
corresponds to equal masses of the primaries, we see in the top left, bottom left, and bottom right corners the stability regions (light grey) of the collinear equilibrium points $L_{2}, L_{1}$, and $L_{3}$, respectively. For lower values of the mass parameter and for the same value of the angular velocity $\omega=0.25$ we observe, in the next frames, that the stability regions of all equilibria become larger and larger and especially in the last frame we see that the triangular equilibrium points $L_{4,5}$ are stable for all values of the parameters $Q_{1}=q_{1}^{1 / 3}$ and $Q_{2}=q_{2}^{1 / 3}$, for which they exist.

In Figures 3, 4, and 5, we show, for comparison reasons, how the corresponding stability regions of the five equilibrium points become, for the specific values of the angular velocity parameter, $\omega=0.5, \omega=1$, and $\omega=2$, by keeping the same values for the mass parameter. Note here that the case $\omega=1$ corresponds to the classical photogravitational restricted three-body problem.

In Figure 3, we observe that for a larger value of the angular velocity parameter $(\omega=0.5)$ the stability regions of the collinear equilibrium points $L_{2}$ and $L_{3}$ are more restricted by the line $Q_{1}+Q_{2}=\omega^{2 / 3}$ (this line cuts both axes to larger values), whereas the stability region for the equilibrium point $L_{1}$ has been increased. In Figure $4, \omega=1$, we see that $Q_{1}+Q_{2}=\omega^{2 / 3}$ cuts the axes $Q_{1}, Q_{2}$ at the points $(1,0)$ and $(0,1)$, respectively, which implies that the collinear points $L_{2}$, $L_{3}$ are no longer stable (for this value of the angular velocity parameter the triangular equilibria coalesce only with the inner equilibrium point $\left.L_{1}\right)$. Finally, in Figure $5(\omega=2)$, we also see that only the triangular and the inner collinear equilibrium points are stable but the stability region for the triangular points has been reduced substantially due to the limitation by the line $Q_{1}+Q_{2}=\omega^{2 / 3}$.

In conclusion, as the value of the parameter $\omega$ increases and up to values $\omega<1$, the stability regions of the collinear equilibrium points $L_{2}$ and $L_{3}$ are restricted more and more by the curve $Q_{1}+Q_{2}=\omega^{2 / 3}$, until these points lose completely their stability for $\omega \geq 1$. For $\omega \geq 1$ the stability of the Lagrangian equilibrium points is transferred only to the inner Eulerian equilibrium point. In all the considered cases, the smaller the value of the mass parameter is, the larger the regions of the stability are.

In Figure 6, we show the stability regions of the triangular equilibrium points and the inner equilibrium point $L_{1}$ in the $(\mu, Q)$ plane in the case where $Q_{1}=Q_{2}=Q$, for various values of the parameter $\omega$. In this figure we do not obtain the stability regions of the outer Eulerian equilibrium points, since it illustrates the entry and exit points of the stability regions of Figures 2, 3, 4, and 5 when we move along the straight line $Q_{2}=Q_{1}$ for all values of the mass parameter. As in the previous figures, we depict the stability region of $L_{1}$ with light grey color and with dark grey we depict the corresponding region of the triangular points. As we see from Figure 6, when we move from the classical photogravitational problem, that is, $\omega=1$, to lower values of the parameter $\omega$, the stability region of the triangular equilibrium points increases, while the stability region of $L_{1}$ decreases due to (12); as we show (12) plays a fundamental role in the area of the corresponding regions for all equilibrium points and in this case takes the special form $Q=\omega^{2 / 3} / 2$ (see the horizontal continuous line, in all frames of this figure). Note here that when $Q_{1}=Q_{2}=Q$ the second of inequalities (32), which arises from the discriminant of the characteristic equation (31), becomes

$$
Q^{4}-36 \mu(1-\mu) \omega^{4 / 3} Q^{2}+9 \mu(1-\mu) \omega^{8 / 3}>0 .
$$

Solving this as a quadratic equation for $q=Q^{2}$ we obtain its discriminant:

$$
-36 \mu(1-\mu) \omega^{8 / 3}[1-36 \mu(1-\mu)]
$$

which is equal to zero when $1-36 \mu(1-\mu)=0$, that is, for the unique acceptable value of the mass parameter:

$$
\mu_{0}=\frac{1}{6}(3-2 \sqrt{2}) \cong 0.028595479 \text {. }
$$

This is a remarkable result for this special case since the curve, which defines the stability region of the triangular equilibrium point, has a minimum value with respect to the mass parameter (see Figure 6) which is independent of the other parameters of the problem; that is, linear stability of triangular equilibria occurs at least for values of the mass parameter which are less than this unique value $\mu_{0}$. This value for the mass parameter has also been found by Schuerman [32] as a terminal value for the stability of the triangular equilibrium points of the photogravitational restricted threebody problem.

\section{Periodic Solutions around Stable Equilibrium Points}

The general solution of (16) is written in the following form:

$$
\begin{aligned}
& \xi(t)=\sum_{i=1}^{4} k_{i} e^{\lambda_{i} t}, \\
& \eta(t)=\sum_{i=1}^{4} m_{i} e^{\lambda_{i} t},
\end{aligned}
$$

where the coefficients $k_{i}, m_{i}, i=1,2,3,4$, may be determined from the initial conditions. In case where all roots of the characteristic equation of the variational matrix (17) are purely imaginary, the motion is bounded and consists of two harmonic motions; the general solution depends on the eigenfrequencies $\sigma_{1}, \sigma_{2}$ and can be written in the following form:

$$
\begin{aligned}
\xi(\tau)= & A_{1} \cos \left(\sigma_{1} t\right)+A_{2} \sin \left(\sigma_{1} t\right)+A_{3} \cos \left(\sigma_{2} t\right) \\
& +A_{4} \sin \left(\sigma_{2} t\right) \\
\eta(\tau)= & B_{1} \cos \left(\sigma_{1} t\right)+B_{2} \sin \left(\sigma_{1} t\right)+B_{3} \cos \left(\sigma_{2} t\right) \\
& +B_{4} \sin \left(\sigma_{2} t\right)
\end{aligned}
$$

where $\sigma_{1}, \sigma_{2}$ are the frequencies corresponding to the long and short periodic orbits, respectively. Therefore, the long 


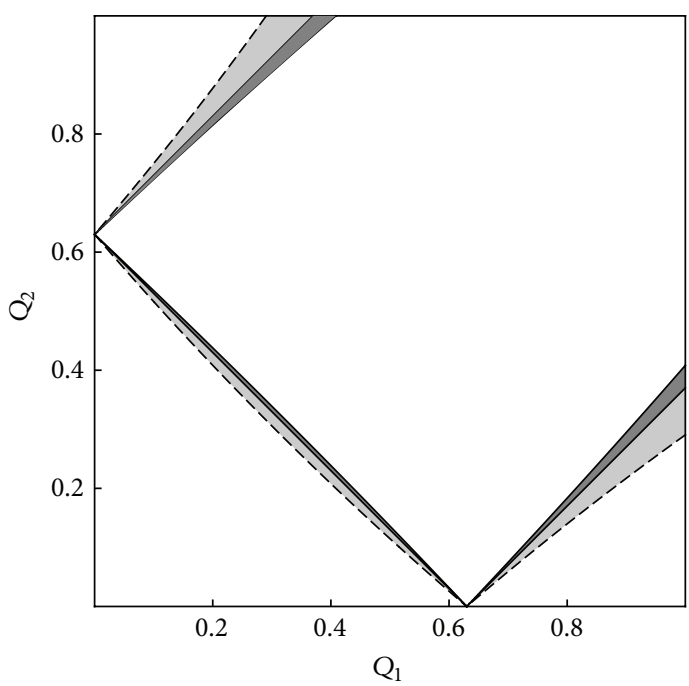

(a)

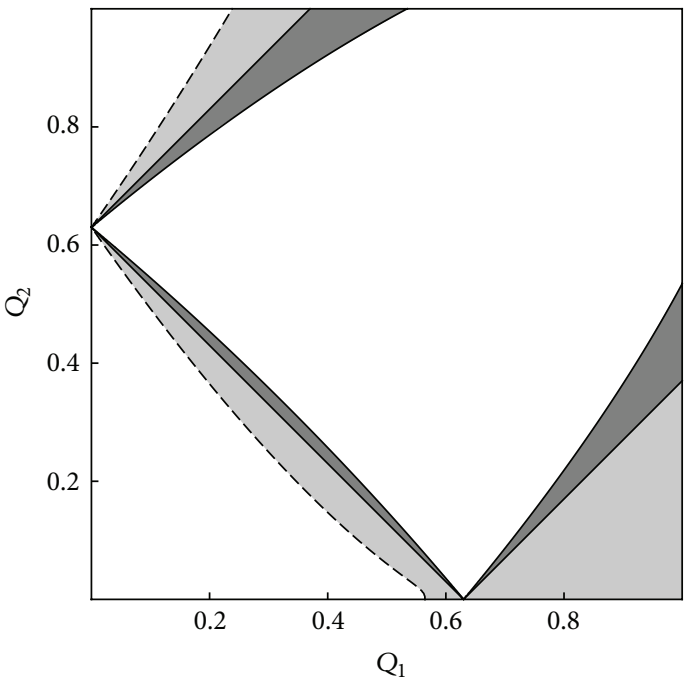

(c)

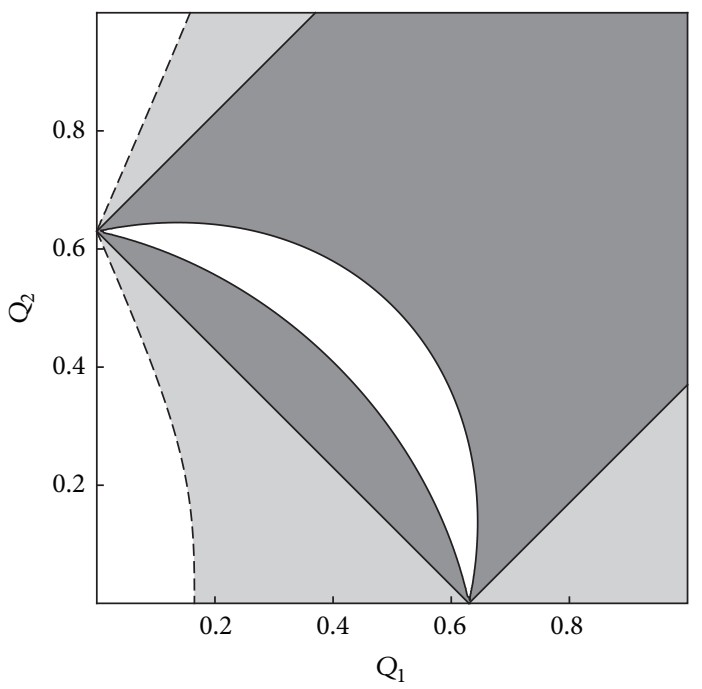

(e)

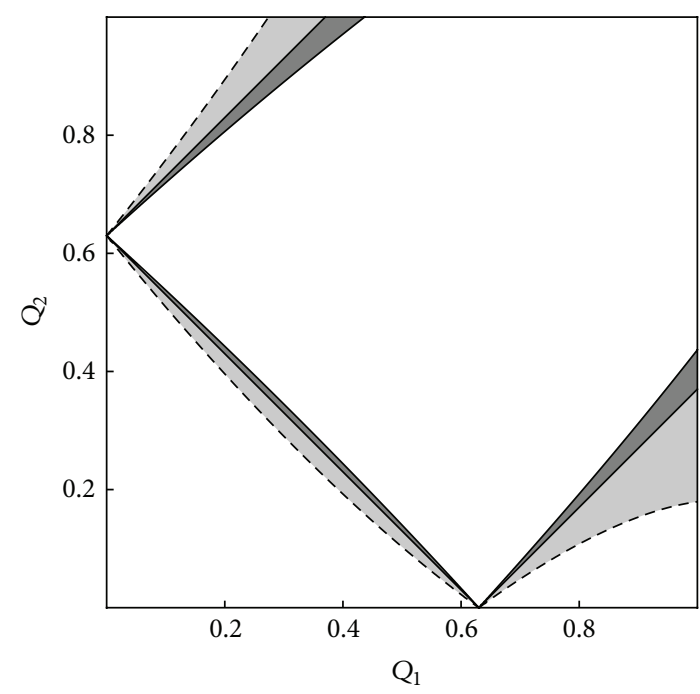

(b)

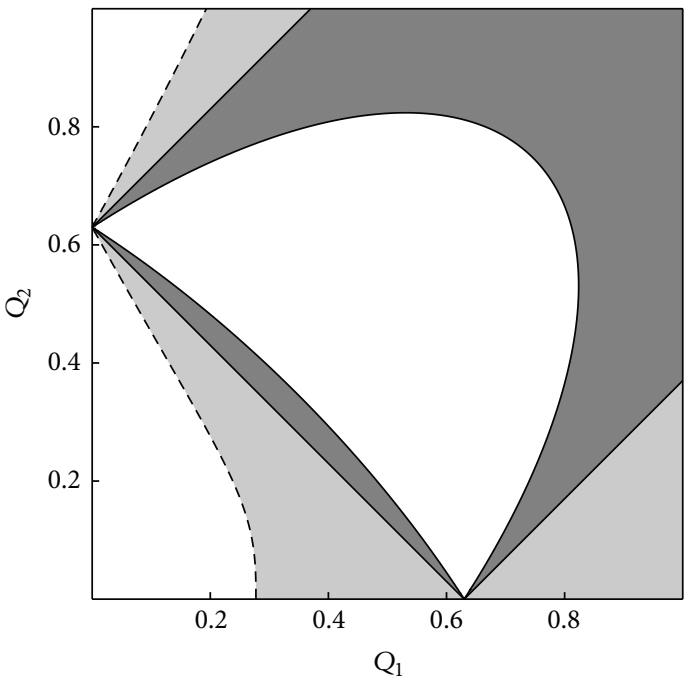

(d)

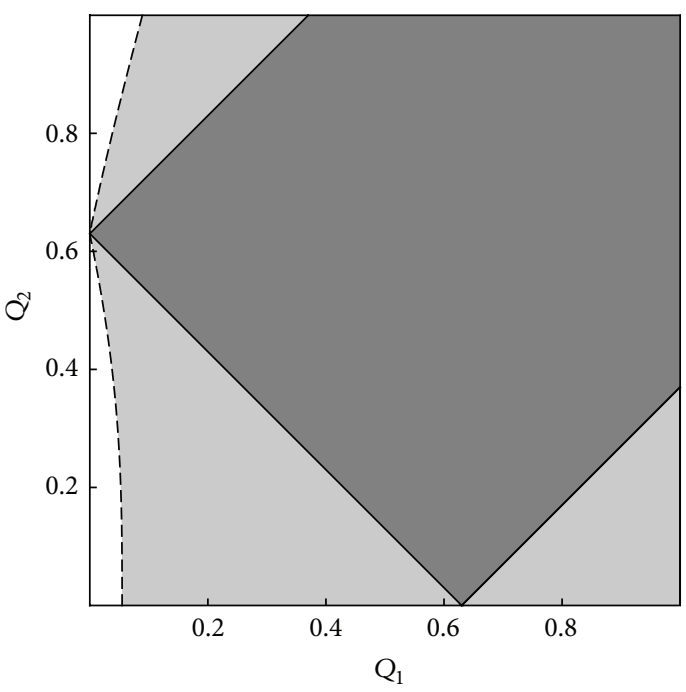

(f)

Figure 3: Stability regions of all equilibrium points in the $\left(Q_{1}, Q_{2}\right)$ plane for $\omega=0.5$ and $\mu=0.5, \mu=0.2, \mu=0.1$, (from (a) to (c)) $\mu=0.05, \mu=0.03$, and $\mu=0.01$ (from (d) to (f)). 


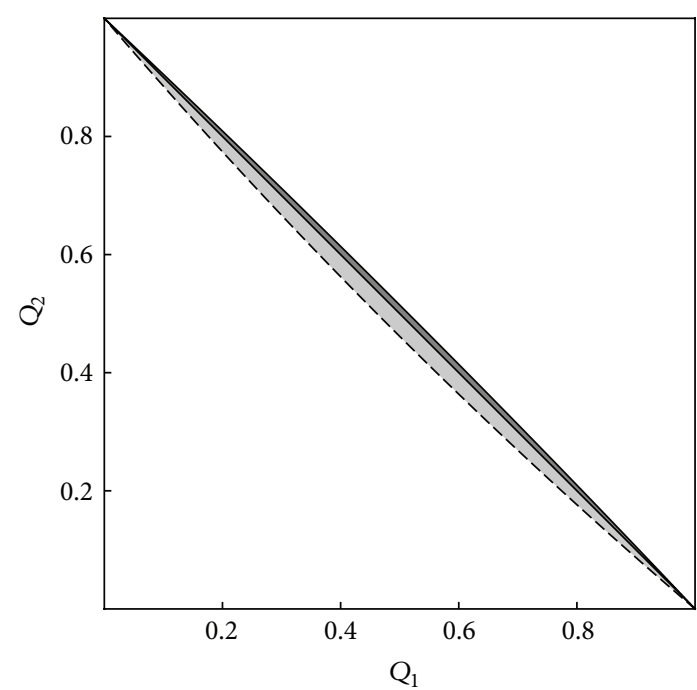

(a)

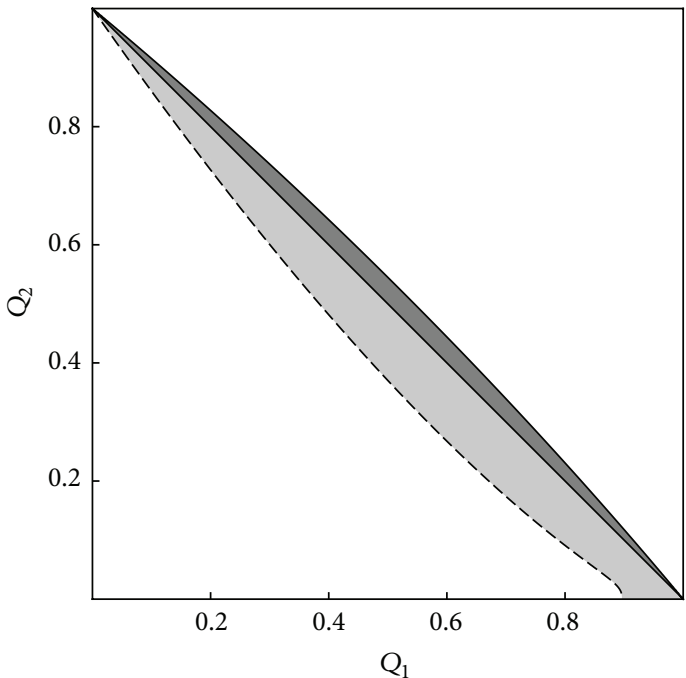

(c)

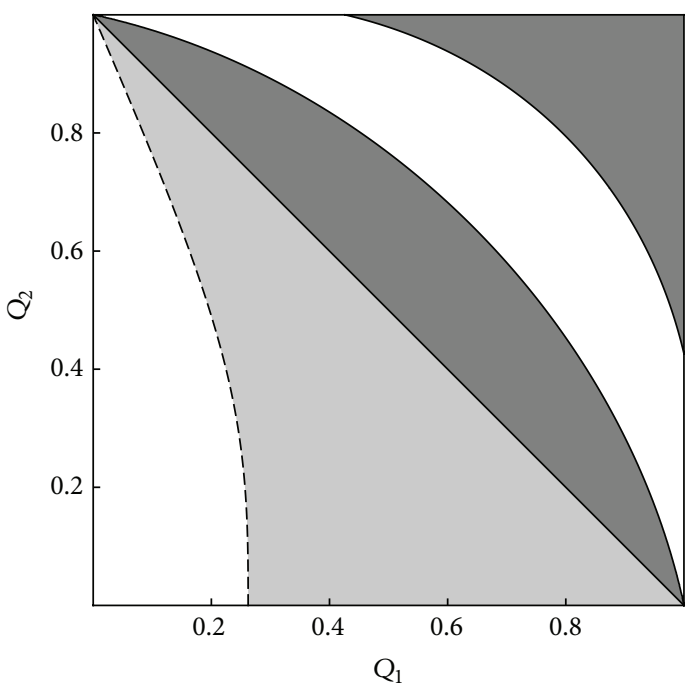

(e)

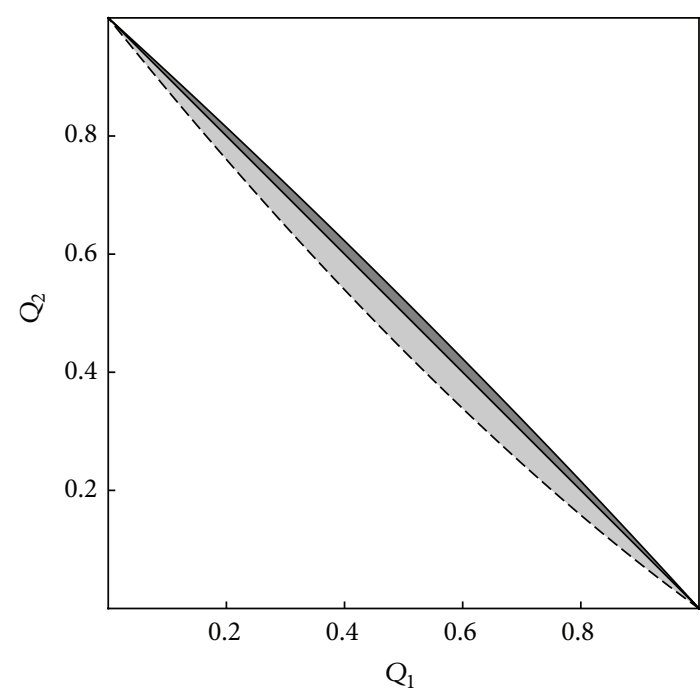

(b)

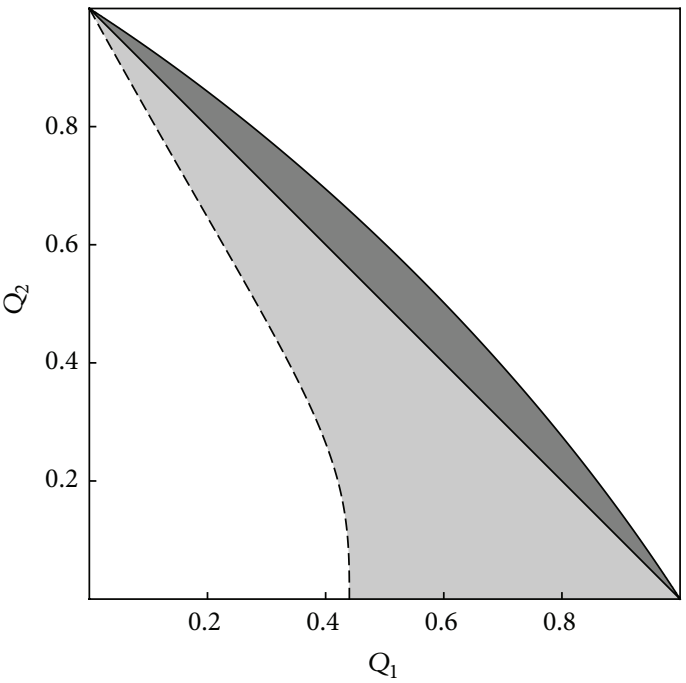

(d)

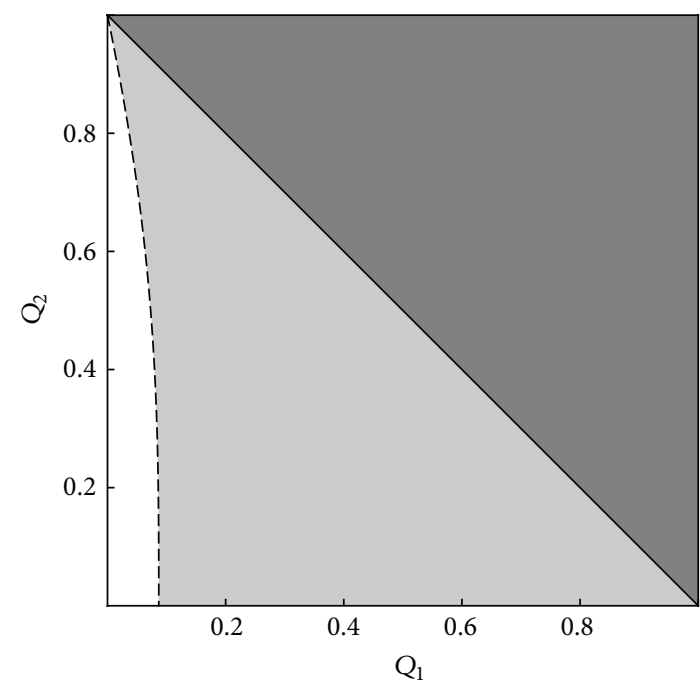

(f)

FIGURE 4: Stability regions of all equilibrium points in the $\left(Q_{1}, Q_{2}\right)$ plane for $\omega=1$ (i.e., the photogravitational restricted three-body problem) and $\mu=0.5, \mu=0.2, \mu=0.1$, (from (a) to (c)) $\mu=0.05, \mu=0.03$, and $\mu=0.01$ (from (d) to (f)). 


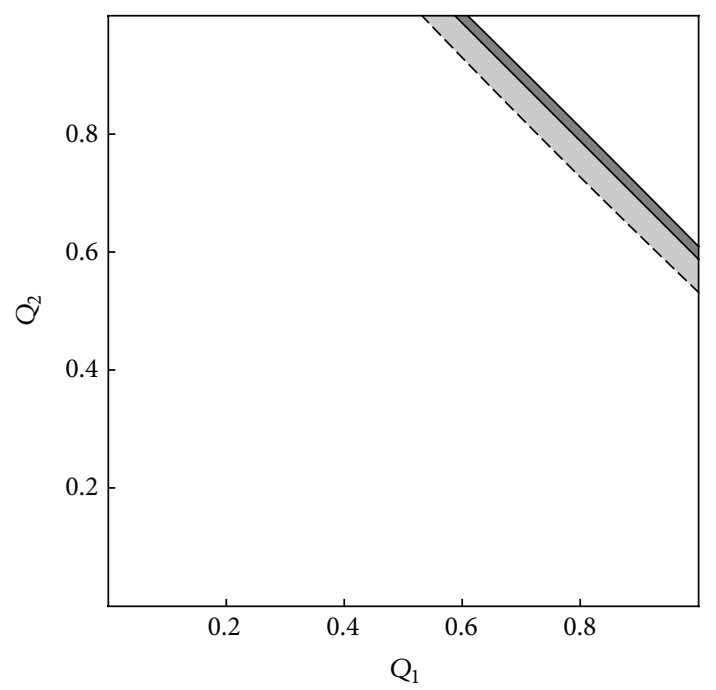

(a)

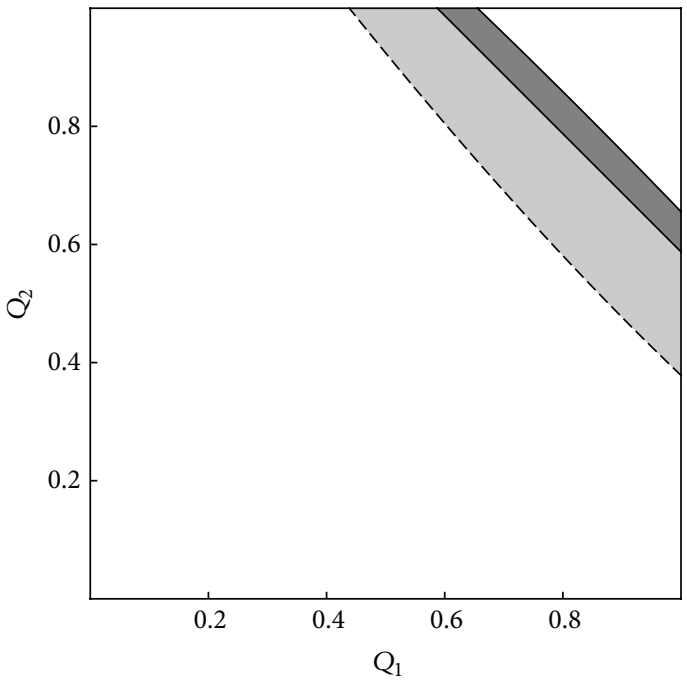

(c)

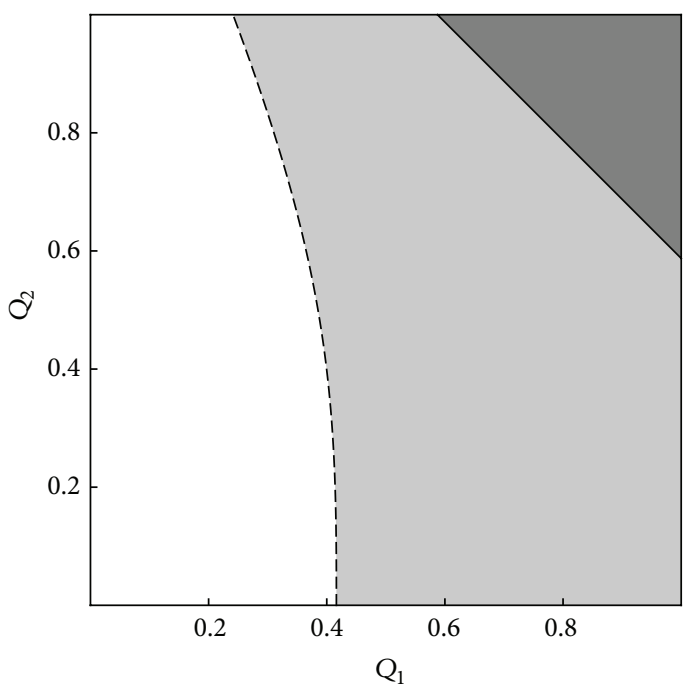

(e)

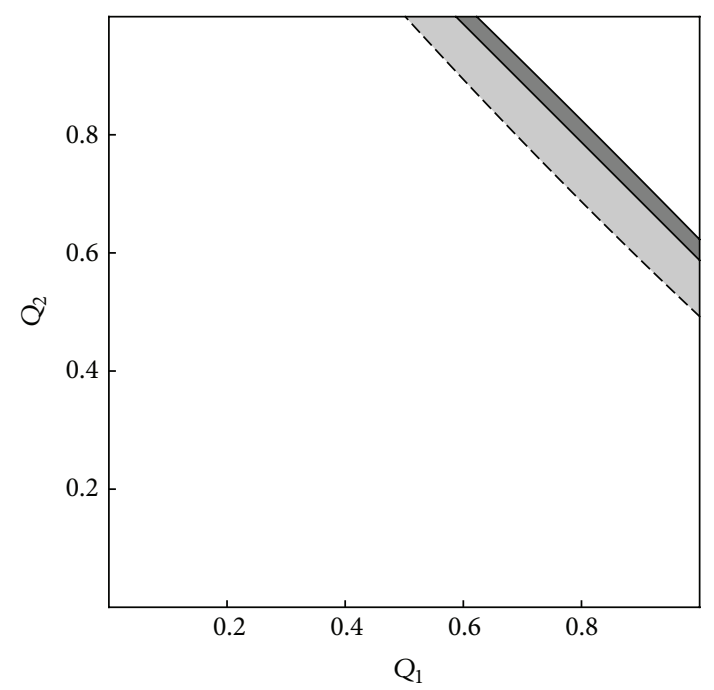

(b)

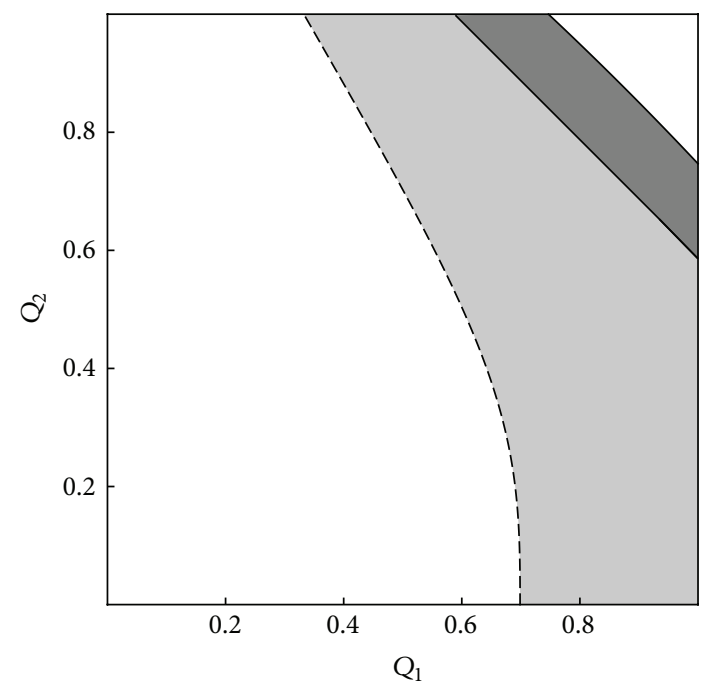

(d)

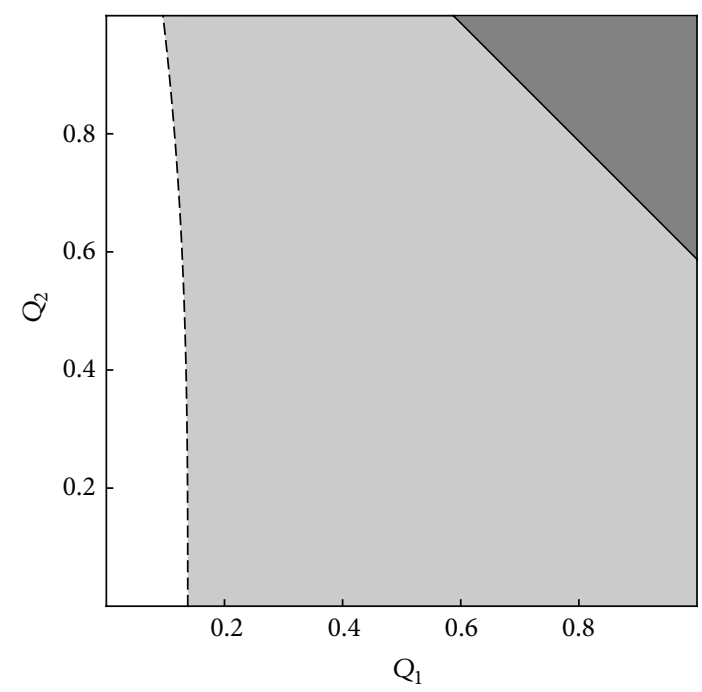

(f)

Figure 5: Stability regions of all equilibrium points in the $\left(Q_{1}, Q_{2}\right)$ plane for $\omega=2$ and $\mu=0.5, \mu=0.2, \mu=0.1$, (from (a) to (c)) $\mu=0.05, \mu=0.03$, and $\mu=0.01$ (from (d) to (f)). 

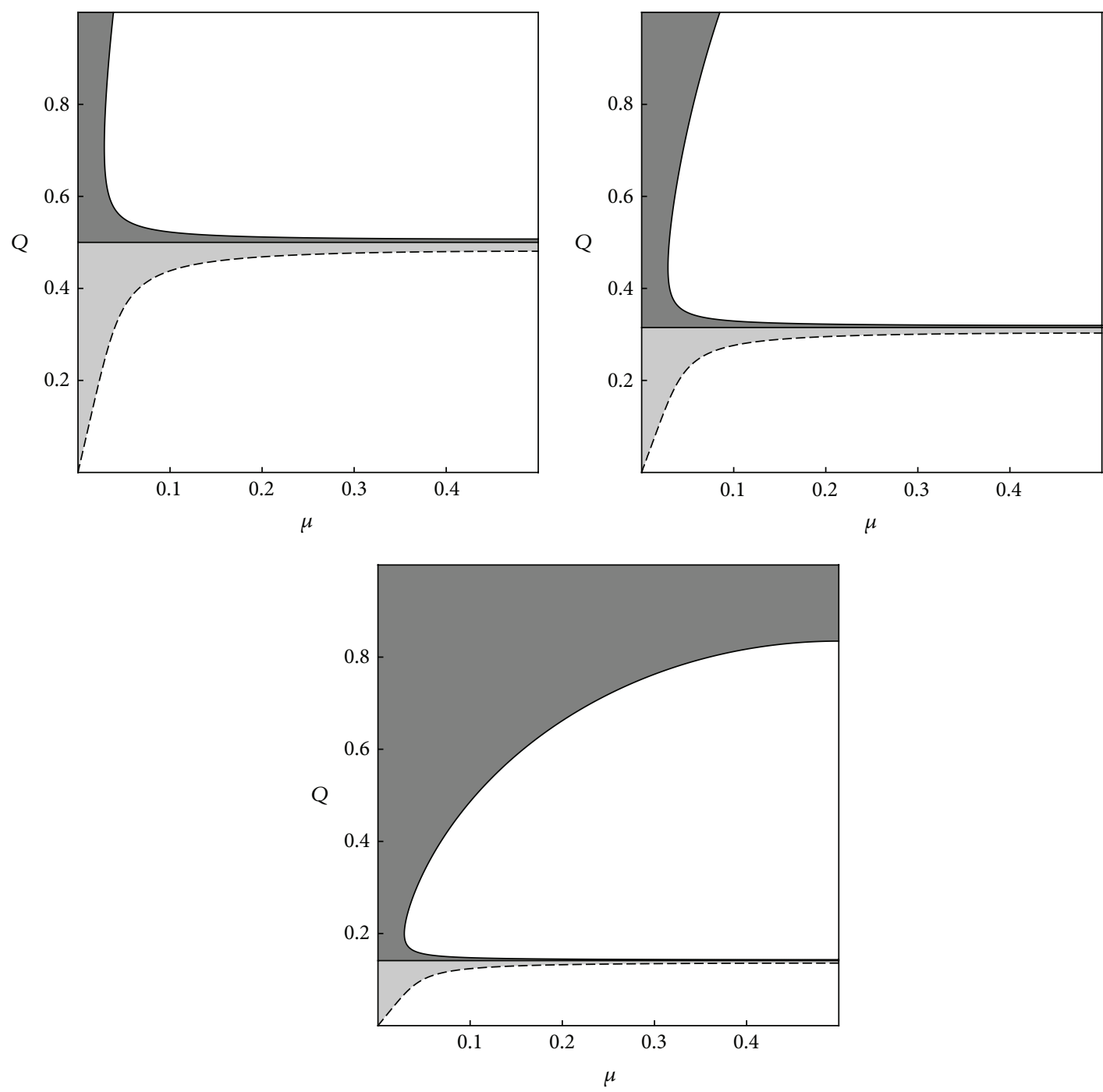

FIGURE 6: Stability regions of the triangular equilibrium points and the inner equilibrium point $L_{1}$ in the $(\mu, Q)$ plane $\left(Q_{1}=Q_{2}=Q\right)$ for $\omega=1, \omega=0.5$, and $\omega=0.15$, respectively.

periodic terms of (39) are those with the coefficients $A_{i}$, $B_{i}, i=1,2$, while the short periodic terms are those with the coefficients $A_{i}, B_{i}, i=3$, 4. Substituting (39) in (16) we obtain for the coefficients $A_{i}$ and $B_{i}, i=1,2,3,4$, that

$$
\begin{aligned}
& A_{1}=\frac{-2 \omega B_{2} \sigma_{1}-\Omega_{x y}^{0} B_{1}}{\sigma_{1}^{2}+\Omega_{x x}^{0}}, \\
& A_{2}=\frac{2 \omega B_{1} \sigma_{1}-\Omega_{x y}^{0} B_{2}}{\sigma_{1}^{2}+\Omega_{x x}^{0}}, \\
& A_{3}=\frac{-2 \omega B_{4} \sigma_{2}-\Omega_{x y}^{0} B_{3}}{\sigma_{2}^{2}+\Omega_{x x}^{0}}, \\
& A_{4}=\frac{2 \omega B_{3} \sigma_{2}-\Omega_{x y}^{0} B_{4}}{\sigma_{2}^{2}+\Omega_{x x}^{0}},
\end{aligned}
$$

$$
\begin{aligned}
& B_{1}=\frac{2 \omega A_{2} \sigma_{1}-\Omega_{y x}^{0} A_{1}}{\sigma_{1}^{2}+\Omega_{y y}^{0}}, \\
& B_{2}=\frac{-2 \omega A_{1} \sigma_{1}-\Omega_{y x}^{0} A_{2}}{\sigma_{1}^{2}+\Omega_{y y}^{0}}, \\
& B_{3}=\frac{2 \omega A_{4} \sigma_{2}-\Omega_{y x}^{0} A_{3}}{\sigma_{2}^{2}+\Omega_{y y}^{0}}, \\
& B_{4}=\frac{-2 \omega A_{3} \sigma_{2}-\Omega_{y x}^{0} A_{4}}{\sigma_{2}^{2}+\Omega_{y y}^{0}} .
\end{aligned}
$$

Recall here that for the collinear equilibrium points $\Omega_{x y}^{0}=\Omega_{y x}^{0}=0$ and all the above formulae are simplified 
giving the following simple relations for the coefficients:

$$
\begin{aligned}
& \frac{A_{2}}{B_{1}}=-\frac{A_{1}}{B_{2}}=\frac{2 \omega \sigma_{1}}{\sigma_{1}^{2}+\Omega_{x x}^{0}}, \\
& \frac{A_{4}}{B_{3}}=-\frac{A_{3}}{B_{4}}=\frac{2 \omega \sigma_{2}}{\sigma_{2}^{2}+\Omega_{x x}^{0}} .
\end{aligned}
$$

Now, for $t=0$, we obtain from (39) the following:

$$
\begin{aligned}
& \xi(0)=A_{1}+A_{3}, \\
& \eta(0)=B_{1}+B_{3}, \\
& \dot{\xi}(0)=A_{2} \sigma_{1}+A_{4} \sigma_{2}, \\
& \dot{\eta}(0)=B_{2} \sigma_{1}+B_{4} \sigma_{2} .
\end{aligned}
$$

In order to determine the long period solution we set $A_{i}=$ $B_{i}=0, i=3,4$, in (39) obtaining

$$
\begin{aligned}
& \xi(t)=\xi(0) \cos \left(\sigma_{1} t\right)+\frac{\dot{\xi}(0)}{\sigma_{1}} \sin \left(\sigma_{1} t\right), \\
& \eta(t)=\eta(0) \cos \left(\sigma_{1} t\right)+\frac{\dot{\eta}(0)}{\sigma_{1}} \sin \left(\sigma_{1} t\right),
\end{aligned}
$$

while for the short periodic solution we set $A_{i}=B_{i}=0$, $i=1,2$, leading to

$$
\begin{aligned}
& \xi(t)=\xi(0) \cos \left(\sigma_{2} t\right)+\frac{\dot{\xi}(0)}{\sigma_{2}} \sin \left(\sigma_{2} t\right), \\
& \eta(t)=\eta(0) \cos \left(\sigma_{2} t\right)+\frac{\dot{\eta}(0)}{\sigma_{2}} \sin \left(\sigma_{2} t\right) .
\end{aligned}
$$

To determine appropriate initial conditions for the numerical integration of the equations of motion (1), for the computation of the family of long periodic orbits, we consider a small orbital parameter $\varepsilon$ so as $\xi(0)=A_{1}=\varepsilon$ and, without loss of generality, $\eta(0)=B_{1}=0$; that is, we start our integration from the transferred $\mathrm{O} x$-axis to any stable equilibrium point. Hence, the first coefficient of (41) gives

$$
A_{2}=\frac{\Omega_{y x}^{0} \varepsilon}{2 \omega \sigma_{1}},
$$

and substituting it in the second coefficient of (41) we also get that

$$
B_{2}=-\frac{4 \omega^{2} \varepsilon \sigma_{1}^{2}+\left(\Omega_{y x}^{0}\right)^{2} \varepsilon}{2 \omega \sigma_{1}\left(\Omega_{y y}^{0}+\sigma_{1}^{2}\right)} .
$$

Accordingly, to determine suitable initial conditions for the numerical computation of the family of short periodic orbits we set $\xi(0)=A_{3}=\varepsilon$ and $\eta(0)=B_{3}=0$ (we also choose to start our integration from the transferred $\mathrm{O} x$-axis), obtaining, thereupon, from the third coefficient of (40) the following:

$$
A_{4}=\frac{\Omega_{y x}^{0} \varepsilon}{2 \omega \sigma_{2}}
$$

and substituting it into the fourth coefficient of (40) one gets the following:

$$
B_{4}=-\frac{4 \omega^{2} \varepsilon \sigma_{2}^{2}+\left(\Omega_{y x}^{0}\right)^{2} \varepsilon}{2 \omega \sigma_{2}\left(\Omega_{y y}^{0}+\sigma_{2}^{2}\right)} .
$$

Therefore, by (43) and (46)-(49) we obtain the corresponding initial conditions:

$$
\begin{aligned}
& \xi(0)=\varepsilon, \\
& \eta(0)=0, \\
& \dot{\xi}(0)=\frac{\Omega_{y x}^{0} \varepsilon}{2 \omega}, \\
& \dot{\eta}(0)=-\frac{\varepsilon\left[4 \omega^{2} \sigma_{i}^{2}+\left(\Omega_{y x}^{0}\right)^{2}\right]}{2 \omega\left(\Omega_{y y}^{0}+\sigma_{i}^{2}\right)},
\end{aligned}
$$

where for $i=1$ we get the initial approximation of a long periodic orbit, while for $i=2$ the corresponding initial approximation of a short periodic orbit is obtained. For small values of the orbital parameter $\varepsilon$ and fixed values of the parameters of the problem, expressions (50) may be used for the numerical determination of the first part of the families of short and long periodic orbits emanating from the stable Eulerian and Lagrangian equilibrium points, which are then continued numerically for the accurate computation of its members with a predetermined accuracy.

4.1. Numerical Results for $q_{1}=q_{2}=\omega=1$ (Classical Problem). We will first focus on the determination of the long period family emanating from the Lagrangian equilibrium point $L_{4}$ (due to the symmetry of the problem with respect to the $\mathrm{O} x$ axis the same results may be obtained for the equilibrium point $L_{5}$ ) for the Sun-Jupiter case of the classical restricted three-body problem, that is, for the mass ratio $\mu=0.00095$, while the remaining parameters of the problem are equal to unity $\left(q_{1}=q_{2}=\omega=1\right)$. Henrard [28] has given a schematic representation of the family evolution and stated that, in the Sun-Jupiter system, the family of long periodic orbits goes to a bifurcation with an orbit of the short period family travelled thirteen times and then it continues and bifurcates with another orbit of the short period family travelled fourteen times. This pattern continues to appear up to, as Henrard conjectured, the long period family's termination on a family of homoclinic orbits. Almost recently, Henrard [33] has presented numerical results for the evolution of this family for $\mu \cong 0.01$ and $\mu=0.0095$. Our aim here is to reproduce that schematic representation of Henrard [28] by precise numerical computations, so as to present the mechanism of the long period family's evolution in more detail. For the vertical stability of the long and short period families, we may mention here the works by Perdios and Zagouras [34] and Hou and Liu [35].

In Figure 7, we present the evolution of this family by showing some of its members in the Oxy-plane. The long period family emanating from the triangular equilibrium 


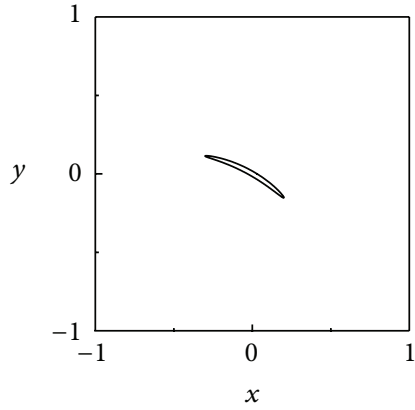

(a)

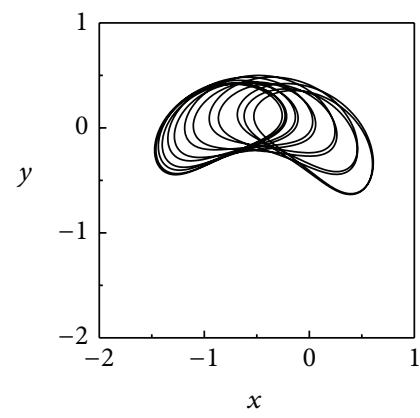

(e)

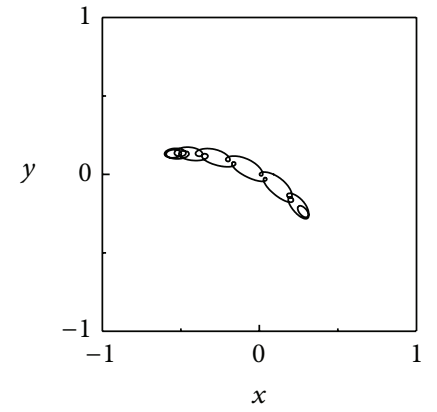

(b)

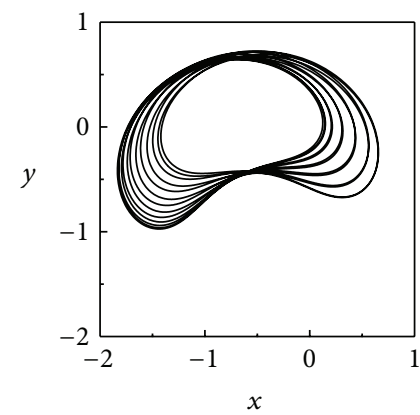

(f)

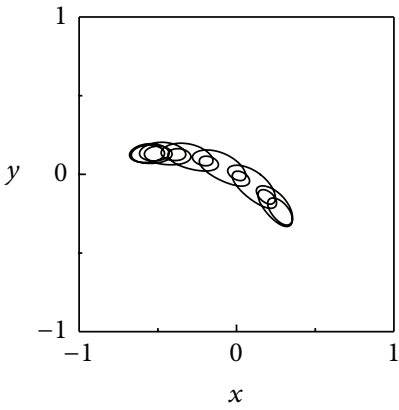

(c)

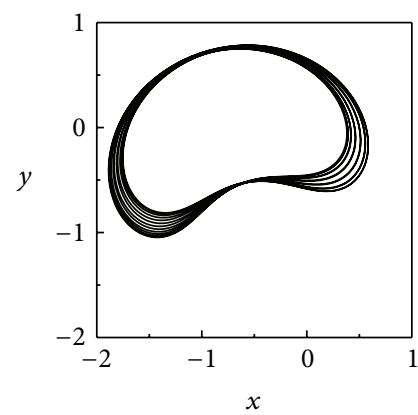

(g)

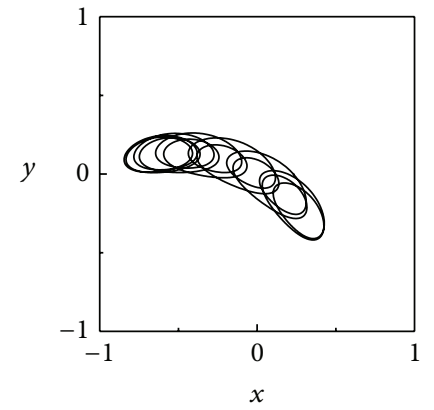

(d)

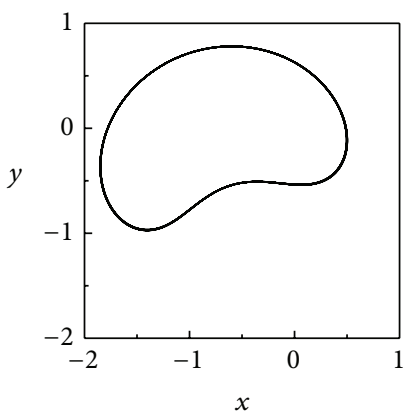

(h)

FIGURE 7: The evolution of the long period family $L_{13}$ by plotting sample periodic orbits. Note that the coordinate system has been transferred to the equilibrium point $L_{4}$.

point $L_{4}$ consists of nonsymmetric periodic orbits with respect to the $\mathrm{O} x$-axis, and its first members are small ovals around the corresponding equilibrium point (Figure 7(a)). Then, its periodic orbits create inner loops (Figure 7(b)) which gradually become tangled (Figures $7(\mathrm{c})$ and $7(\mathrm{~d})$ ). Afterwards, these loops start unraveling (Figures $7(\mathrm{e})-7(\mathrm{~g})$ ) and finally all loops coincide to a simple nonsymmetric periodic orbit, which is the bifurcation orbit of the short period family travelled thirteen times (Figure $7(\mathrm{~h})$ ). We denote this part of the long period family by $L_{13}$.

In Figure 8, we show the remarkable next evolution of the family of long periodic orbits. We observe that as the family continues, its orbits unfold gradually (Figures $8(a)$ and 8(b)), while its next orbits are tangled (Figures 8(c) and $8(\mathrm{~d})$ ) which gradually unravel (Figure $8(\mathrm{e})$ ) and in the sequel they form small ovals around the corresponding triangular equilibrium point (Figure $8(\mathrm{f})$ ). Thereafter, the long periodic orbits start again to curl (Figures $8(\mathrm{~g})-8(\mathrm{i})$ ); then the tangled orbits progressively unravel (Figure $8(\mathrm{j})$ ) and their loops tend to coincide to a simple nonsymmetric periodic orbit around $L_{4}$ (Figure $8(\mathrm{k})$ ) until the long period family bifurcates with the short period family to an orbit travelled fourteen times (Figure $8(\mathrm{l})$ ). We denote this part of the long period family by $L_{13}^{14}$. This pattern of orbits of branch $L_{13}^{14}$ also appears for the next part of the long period family, with the difference that now the new branch goes on an orbit of the short period family travelling it fifteen times. Accordingly, we denote this branch by $L_{14}^{15}$ (we do not present the corresponding orbits due to their similarity with the orbits of Figure 8). For the next branch of the long period family the same behavior has been observed and following the same notation we denote it by $L_{15}^{16}$.

An alternative illustration of the evolution of the long period family is also shown in Figure 9, where we present the projection of its characteristic curve in the parametric plane $\left(T, x_{0}\right)$ of the space of initial conditions $\left(x_{0}, y_{0}=\right.$ $\left.0, \dot{x}_{0}, \dot{y}_{0} ; T\right)$, where $T$ is the period. Note here that the ordinate is zero since we have transferred the coordinate system to $L_{4}$ and for the numerical computation of the corresponding family we always seek a crossing with the new $\mathrm{O} x$-axis. Also, in this figure, we denote by $S_{i}, i=$ $13,14,15,16$, the bifurcation orbits of the short period family, where the subscript denotes the times that the orbit of the long family travels the corresponding orbit of the short family at the bifurcation point. Figures 9 (b) $-9(d)$ are appropriate magnifications of parts of Figure 9(a). As we see, in frame (a), the long period family $\left(L_{13}\right)$ emanates from the triangular equilibrium point $L_{4}$ and bifurcates with the short period family at the periodic orbit $S_{13}$, which means that this orbit is travelled thirteen times by the corresponding periodic orbit of the long family (see Figure 9(b) for this junction). After this bifurcation point the long period family continues as $L_{13}^{14}$ and bifurcates the short period family at the orbit $S_{14}$; that is, this orbit is travelled fourteen times (see frame (c) for this junction). It then continues as $L_{14}^{15}$ and goes to the bifurcation orbit $S_{15}$ travelling it fifteen times (see frame (d) for this junction) and continues as $L_{15}^{16}$. This pattern of evolution of the long period family seems to continue but we have stopped 


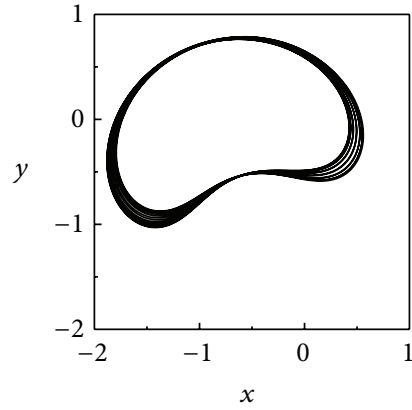

(a)

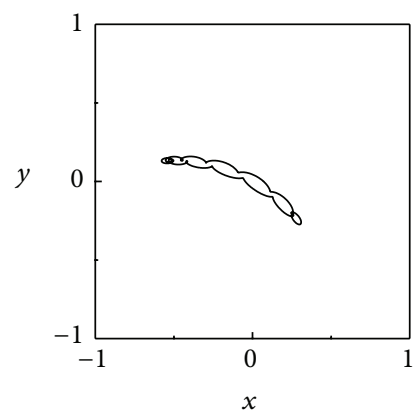

(e)

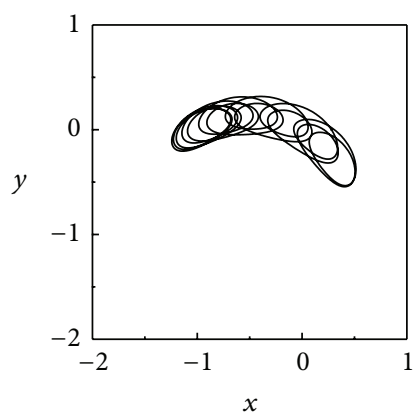

(i)

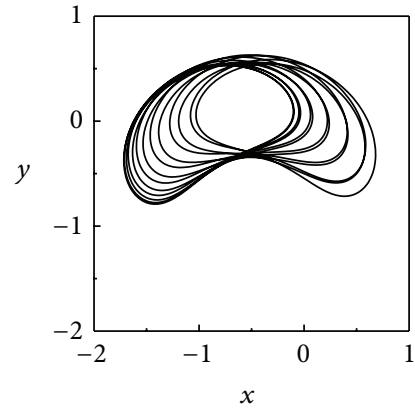

(b)

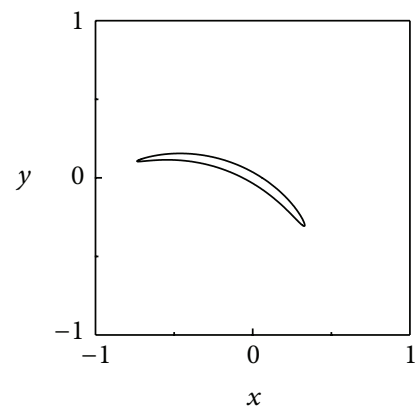

(f)

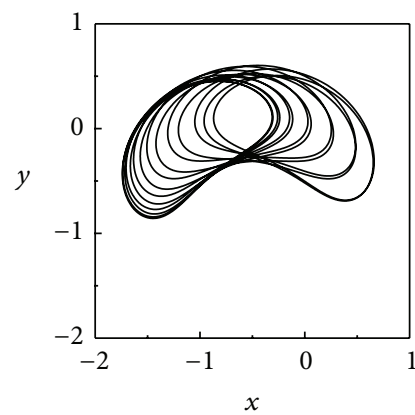

(j)

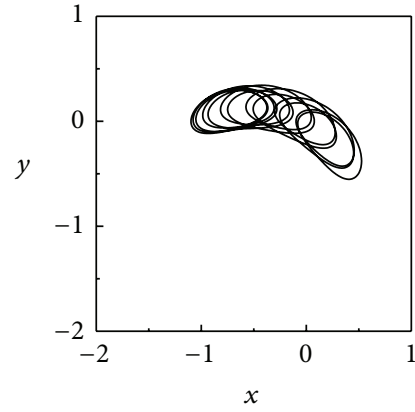

(c)

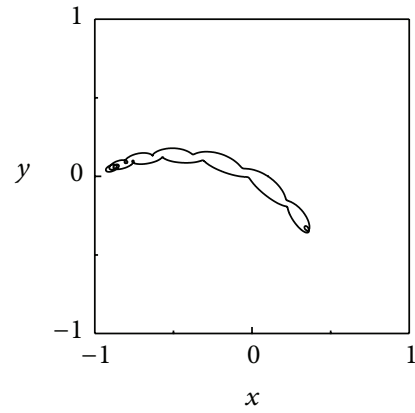

(g)

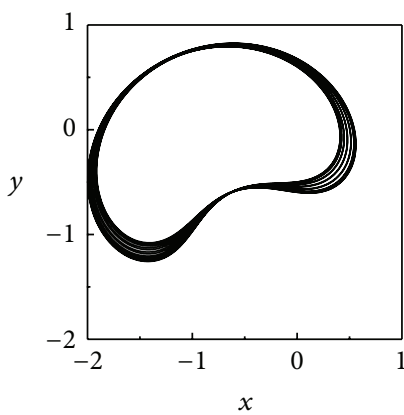

(k)

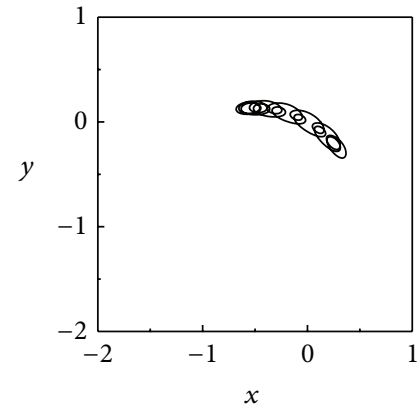

(d)

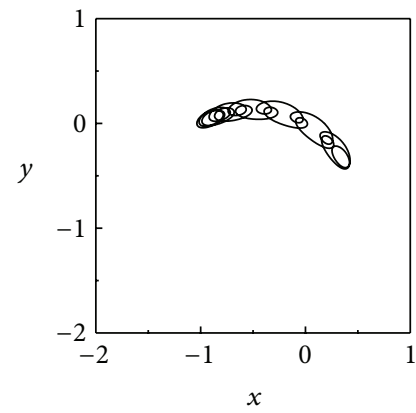

(h)

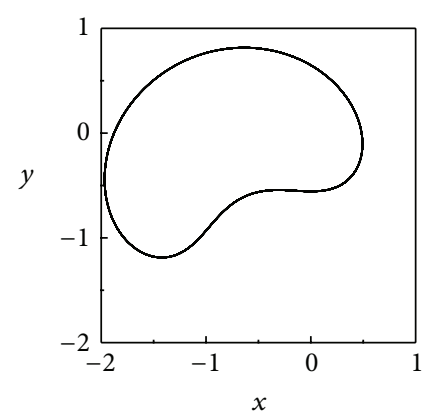

(l)

FIGURE 8: The evolution of the branch $L_{13}^{14}$ of the long period family by plotting sample periodic orbits. Note that the coordinate system has been transferred to the equilibrium point $L_{4}$.

TABLE 1: Sample members of the long period family emanating from the triangular equilibrium point $L_{4}$ for the Sun-Jupiter system of the classical restricted three-body problem $(\mu=0.00095)$.

\begin{tabular}{cccccccc}
\hline & $T$ & $x_{0}$ & $\dot{x}_{0}$ & $\dot{y}_{0}$ & $C$ & $s_{h}$ \\
\hline$L_{13}$ & 81.965981 & 0.2851750 & 0.1855400 & -0.2593142 & 2.9747462 & 1.023708 & 0.951895 \\
$L_{13}^{14}$ & 88.312060 & 0.1696324 & 0.1210896 & -0.1151430 & 2.9964843 & 1.021820 & 0.929480 \\
$L_{14}^{15}$ & 94.548562 & 0.3197985 & 0.1679201 & -0.3395816 & 2.9546957 & 5.590865 \\
$L_{15}^{16}$ & 100.870333 & 0.2765249 & 0.1515870 & -0.2739704 & 2.9733912 & 7.641533 & 0.932739 \\
\hline
\end{tabular}

our numerical computations at this point. Figure 9 shows that a connection of the bifurcation orbits $S_{i}, i=13,14,15,16$, of the short period family exists through the characteristic curve of the long period family. In Figure 10, we present this connection in the parametric plane $\left(x_{0}, \dot{x}_{0}\right)$. For a detailed study on the evolution of the short period family for the SunJupiter system we refer to Goodrich [36].
In Table 1, we present initial conditions of representative periodic orbits of the long period family. In particular, for each nonsymmetric periodic orbit, we give its period $T$, its abscissa $x_{0}$ on the Ox-axis of the transferred to the triangular equilibrium point coordinate system, the velocity components $\dot{x}_{0}$ and $\dot{y}_{0}$, the value of the Jacobi constant $C$, and the stability parameters $s_{h}$ and $s_{v}$. Note here that, according to 


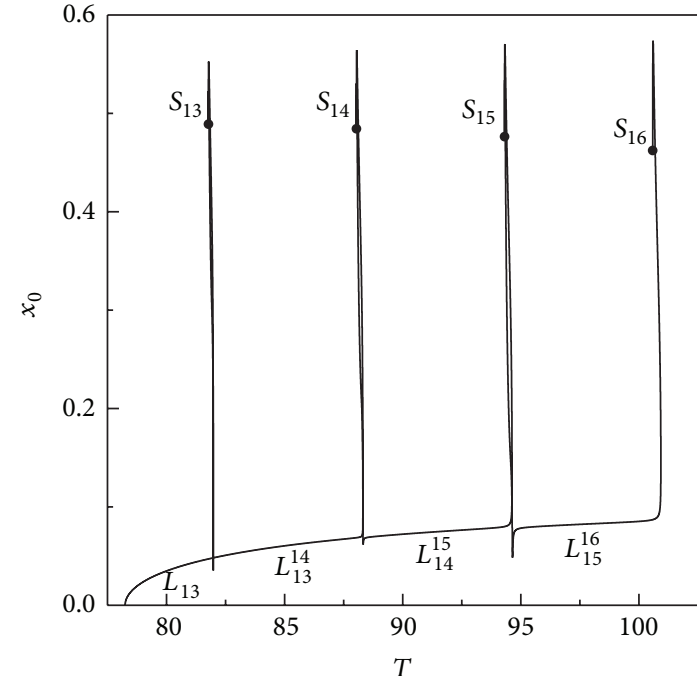

(a)

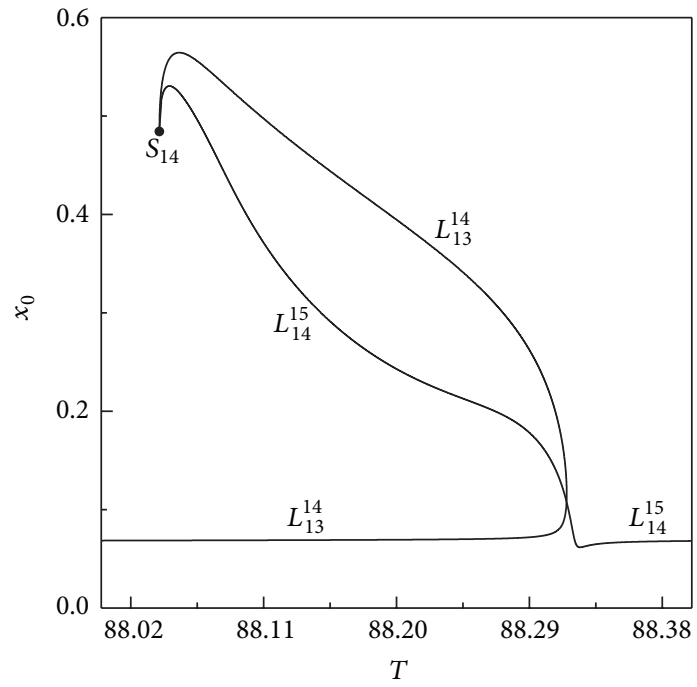

(c)

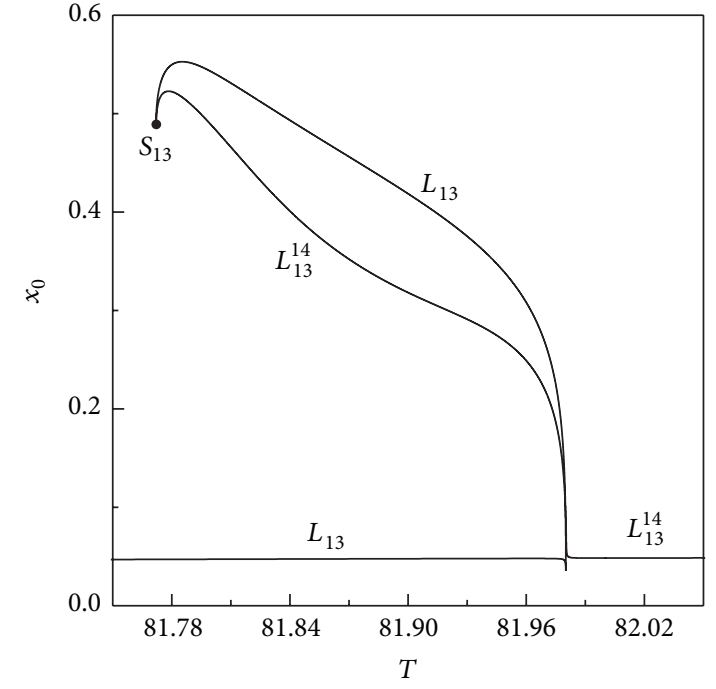

(b)

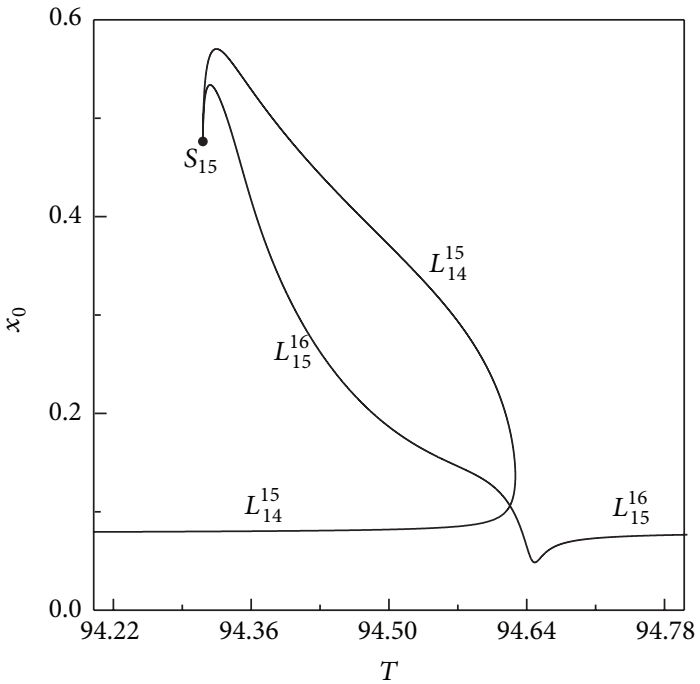

(d)

FIGURE 9: (a) Projection of the characteristic curve of the long period family in the ( $\left.T, x_{0}\right)$ plane. (b), (c), and (d) appropriate magnifications of certain parts of (a).

Hénon [37, 38], a periodic orbit is stable if both $\left|s_{h}\right|<1$ and $\left|s_{v}\right|<1$ hold where

$$
\begin{gathered}
s_{h}=\frac{a_{h}+d_{h}}{2}, \\
s_{v}=\frac{a_{v}+d_{v}}{2},
\end{gathered}
$$

with $a_{h}, d_{h}$ and $a_{v}, d_{v}$ being the horizontal and vertical stability parameters, correspondingly. If only one of these inequalities hold, the orbit is considered horizontally or vertically stable, respectively (for the computation of the horizontal stability, see also the formulae given by Markellos [39]). In the case of symmetric periodic orbits $a_{h}=d_{h}$ and $a_{v}=d_{v}$, obtaining thus $s_{h}=a_{h}$ and $s_{v}=a_{v}$.
4.2. Numerical Results for $q_{1}, q_{2}, \omega \neq 1$. We will now proceed to incorporate the other parameters of the problem and compute the corresponding long and short period families. The stability analysis of the equilibrium points, in Section 3, shows that additionally to the triangular equilibria these families may also be found at the collinear equilibrium points. Therefore, for our next results, the values of the parameters have been arbitrarily chosen but such that the corresponding equilibrium points are stable. We may mention here the work by Papadakis [40], in which periodic motions around the triangular equilibrium points of the Chermnykh's problem for the Sun-Jupiter mass distribution were studied, as well as the corresponding short and long period families emanating from the inner equilibrium point $L_{1}$ for the critical value of the angular velocity parameter $\omega=2 \sqrt{2}$ at which the triangular equilibrium points disappear by coalescing with 


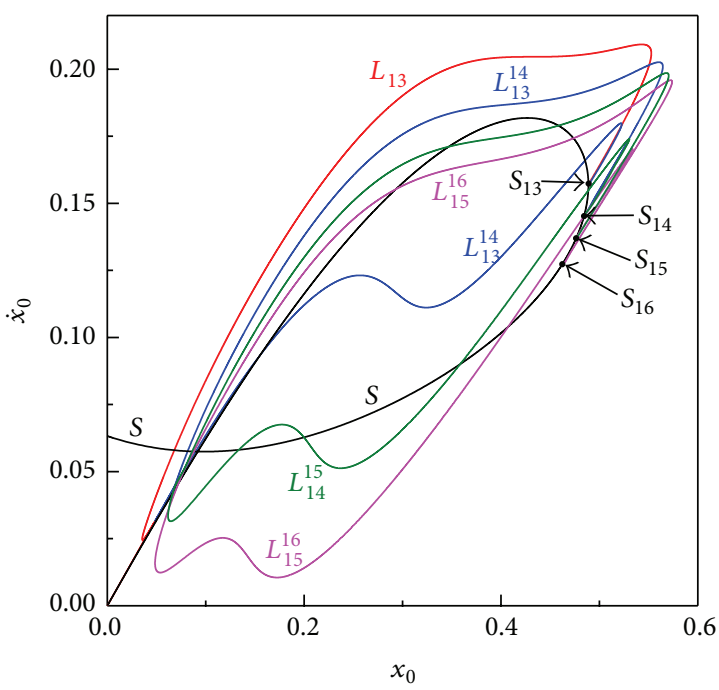

(a)

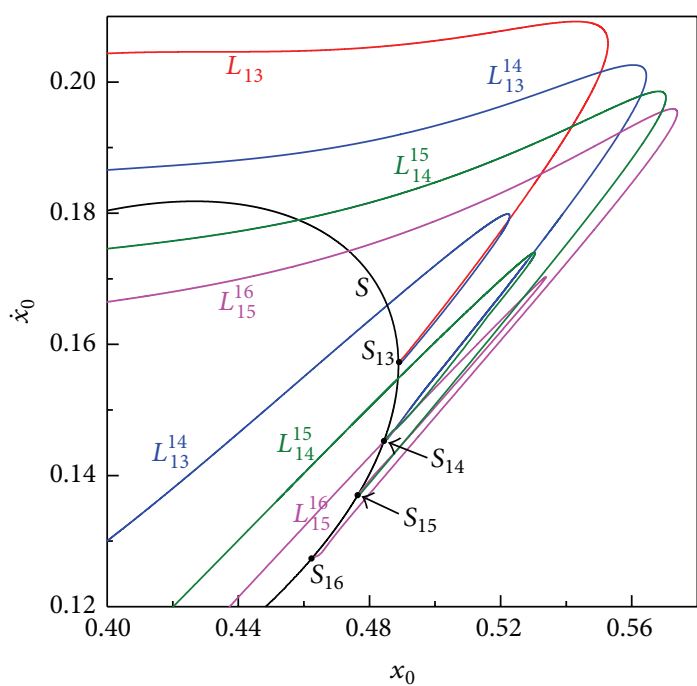

(b)

Figure 10: (a) Projection of the characteristic curve of the long period family in the $\left(x_{0}, \dot{x}_{0}\right)$ plane. (b) Magnification of part of the left frame. In both frames, $S$ denotes the short period family.

TABLE 2: Sample members of the long period family emanating from the triangular equilibrium point $L_{4}$ for $q_{1}=0.5, q_{2}=0.8$, $\omega=1.5$, and $\mu=0.00095$.

\begin{tabular}{cccccccc}
\hline & $T$ & $x_{0}$ & $\dot{x}_{0}$ & $\dot{y}_{0}$ & $C$ & $s_{h}$ \\
\hline$L_{12}$ & 50.433206 & 0.1294818 & 0.1565572 & -0.2214219 & 2.4608218 & 0.817029 & 0.958225 \\
$L_{12}^{13}$ & 54.583313 & 0.1250745 & 0.1515822 & -0.2084973 & 2.4639318 & 0.634769 & 0.966236 \\
$L_{13}^{14}$ & 58.728922 & 0.1157852 & 0.1421300 & -0.1878419 & 2.4669604 & 1.401536 & 0.975416 \\
\hline
\end{tabular}

TABLE 3: Sample members of the short period family emanating from the triangular equilibrium point $L_{4}$ for $q_{1}=0.5, q_{2}=0.8, \omega=1.5$, and $\mu=0.00095$.

\begin{tabular}{lccccc}
\hline$T$ & $x_{0}$ & $\dot{x}_{0}$ & $\dot{y}_{0}$ & $C$ & $s_{h}$ \\
\hline 4.206499 & 0.0220000 & 0.0239493 & -0.0423981 & 2.4744822 & 1.894299 \\
4.205279 & 0.1520000 & 0.1352379 & -0.3200771 & 2.4361058 & 1.848432 \\
4.197186 & 0.4156000 & 0.2395543 & -1.0010119 & 2.0169264 & 1.607336 \\
4.187397 & 0.2010095 & 0.0259248 & -1.2868579 & 0.9614449 & -0.581246 \\
\hline
\end{tabular}

$L_{1}$ transferring to it their stability. However, the addition in the Chermnykh's problem of the new parameters $q_{1}$ and $q_{2}$, corresponding to the new force of the radiation pressure, allows the existence of short and long period families at all collinear equilibrium points.

With regard to the triangular equilibrium points we have determined the corresponding families for the mass ratio $\mu=$ 0.00095, as in Section 4.1, while the values of the remaining parameters are $q_{1}=0.5, q_{2}=0.8$, and $\omega=1.5$, respectively. The corresponding diagrams are shown in Figure 11. As we see from this figure, the evolution of the long period family follows the same mechanism as in the classical problem, but now the corresponding family goes to a bifurcation with an orbit of the short period family travelled twelve times and then it continues and bifurcates with another orbit of the short period family travelled thirteen times and so on. The asymmetric periodic orbits of the family fold and unfold as in the classical case (see Figures 7 and 8); therefore, we do not show their figures here. The characteristic curve of the short period family is shown in Figure 11(d) (black curve marked by $S$ ) and we note here that, as in the classical problem (see, e.g., Deprit et al. [41]), it terminates at a symmetric periodic orbit belonging to the Lyapunov family emanating from the equilibrium point $L_{3}$ (see Figure 12(a) for this bifurcation), at which it encounters with the corresponding short period family emanating from the other triangular equilibrium point $L_{5}$. Sample members of the short period family are presented in Figure 12(b) in which the bifurcation orbit is shown blue.

In Tables 2 and 3, we give initial conditions of sample members of the long and short period families, respectively. Specifically, for each nonsymmetric periodic orbit of these two families, we give the full period $T$, the abscissa $x_{0}$ on the new $\mathrm{O} x$-axis located at the triangular equilibrium point, the velocity components $\dot{x}_{0}, \dot{y}_{0}$, and the value of the Jacobi 


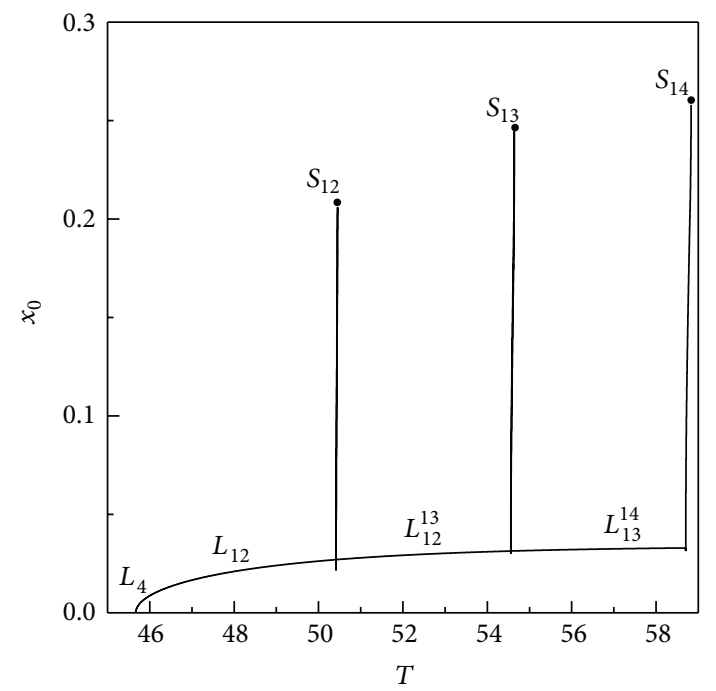

(a)

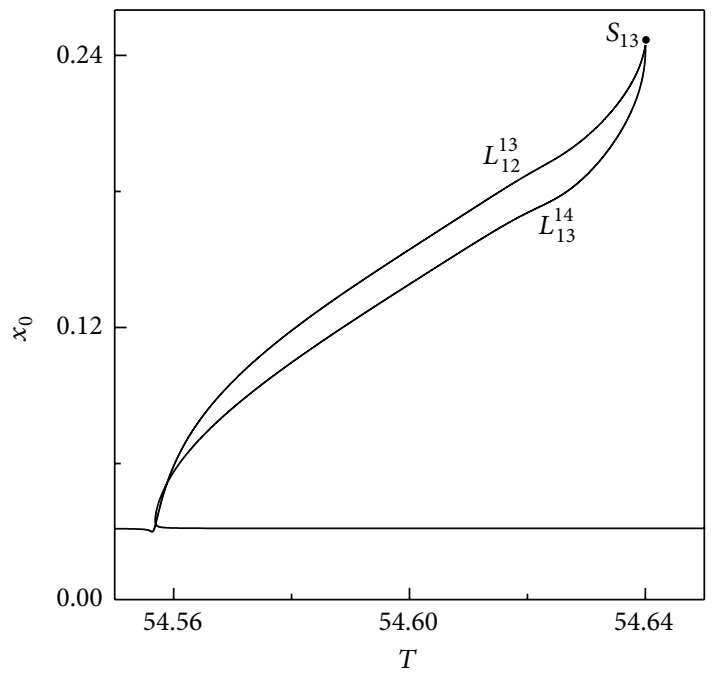

(c)

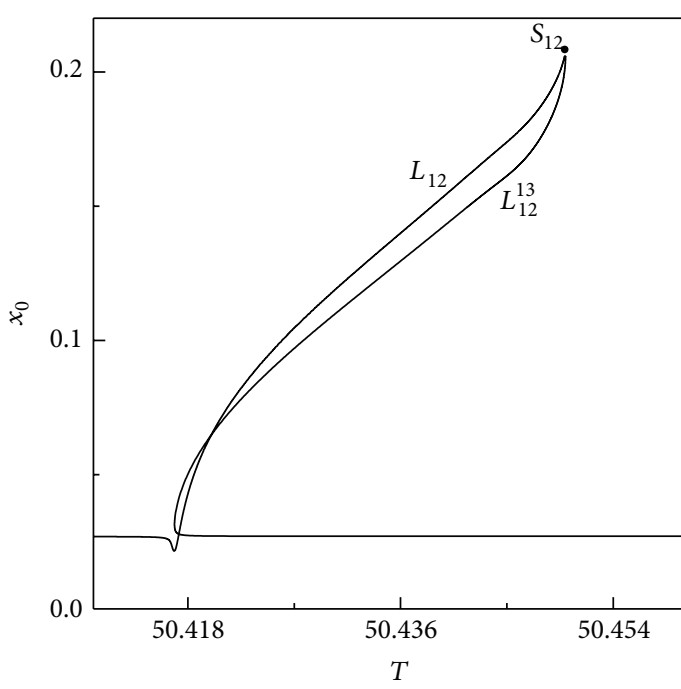

(b)

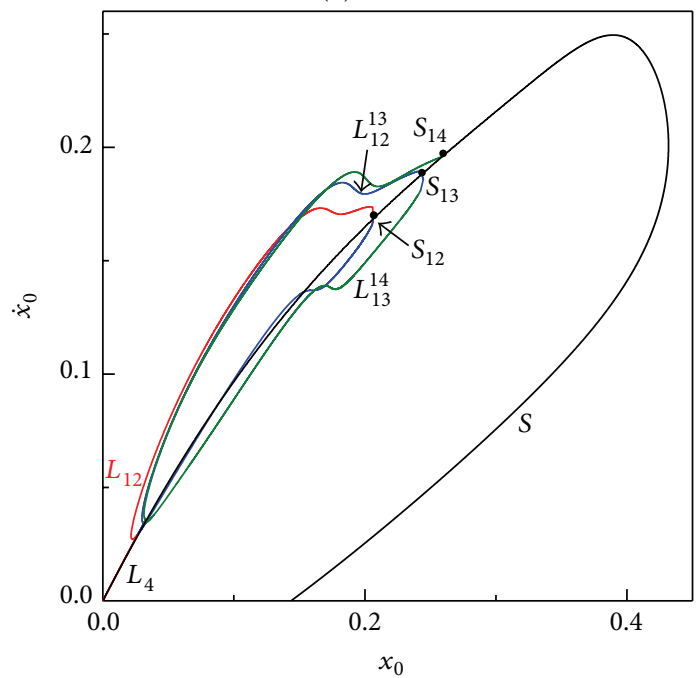

(d)

FIGURE 11: Projection of the characteristic curve of the long period family for the parameter values $q_{1}=0.5, q_{2}=0.8, \omega=1.5$, and $\mu=0.00095$ (a) in the (T, $\left.x_{0}\right)$ plane; (b), (c) magnifications of certain parts of the first frame showing the evolution in detail and (d) in the $\left(x_{0}, \dot{x}_{0}\right)$ plane.

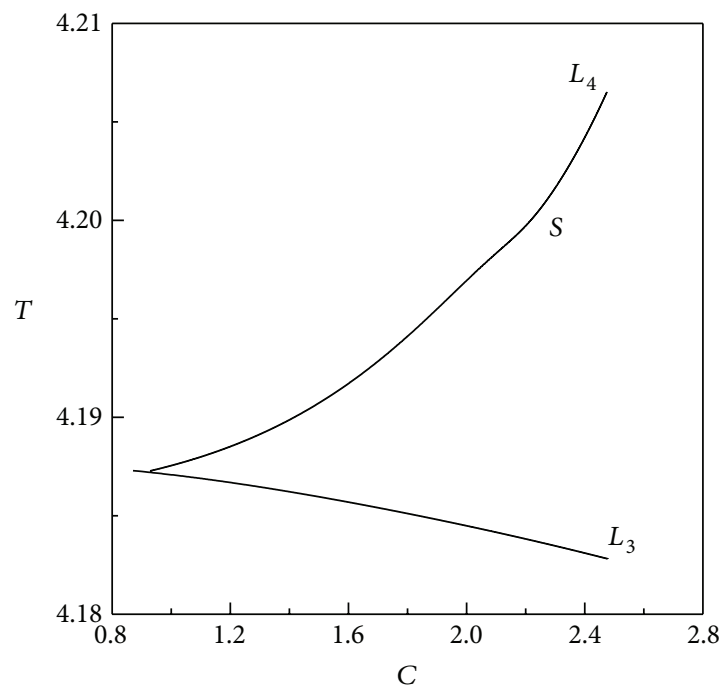

(a)

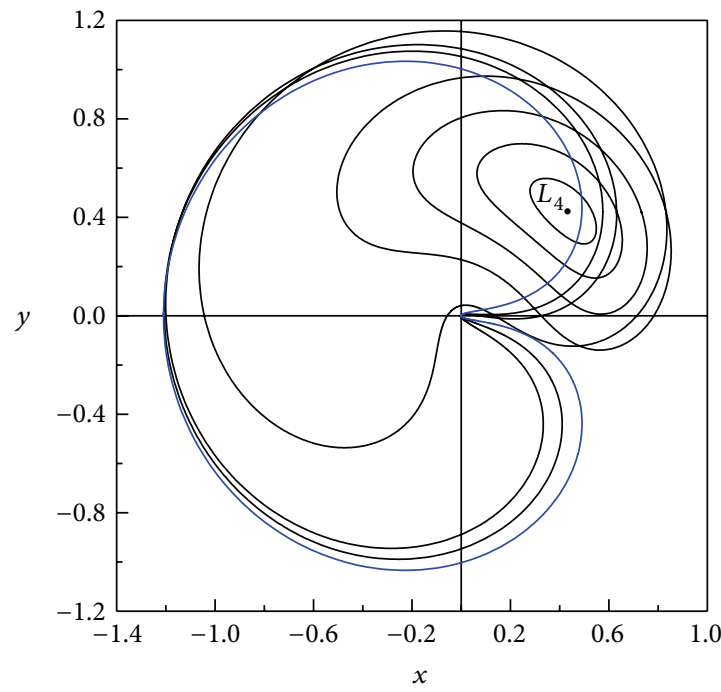

(b)

FIGURE 12: (a) The bifurcation of the short period family with the Lyapunov family emanating from the collinear equilibrium point $L_{3}$. (b) Typical members of the short period family. The bifurcation orbit is shown blue. 


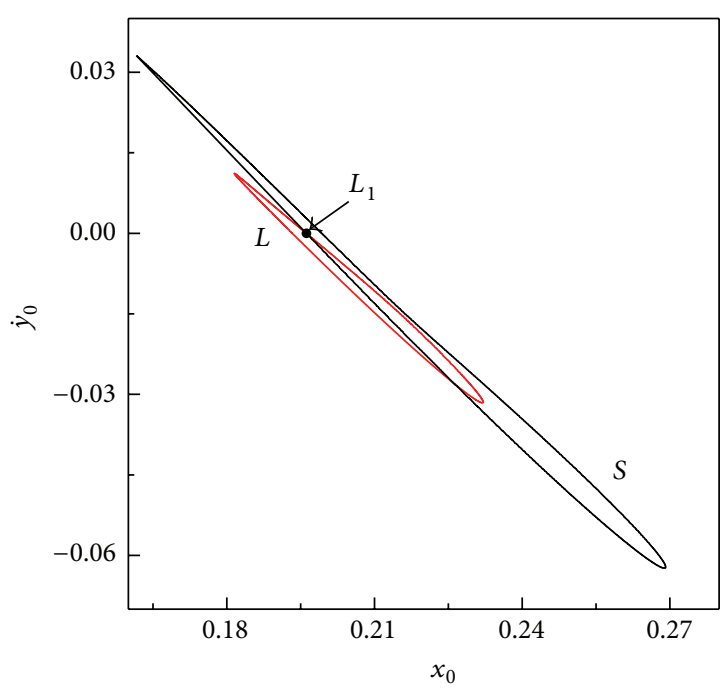

(a)

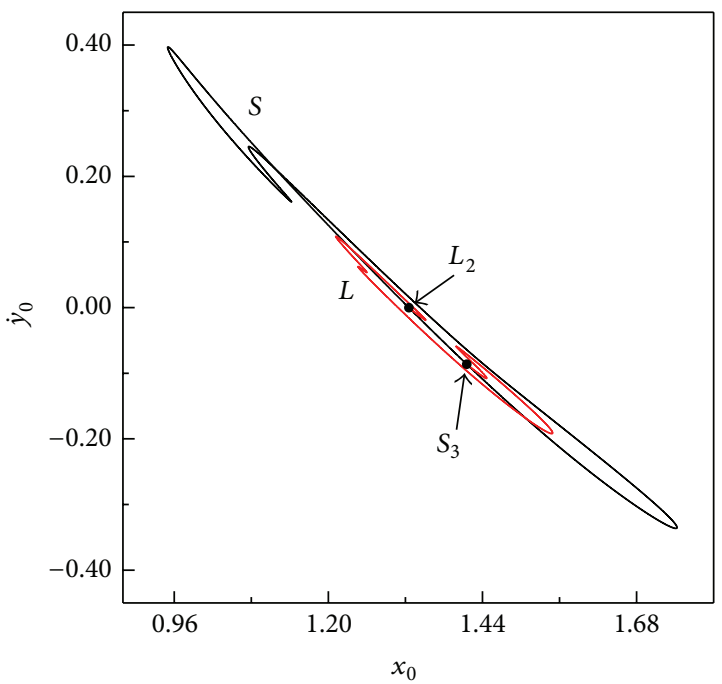

(b)

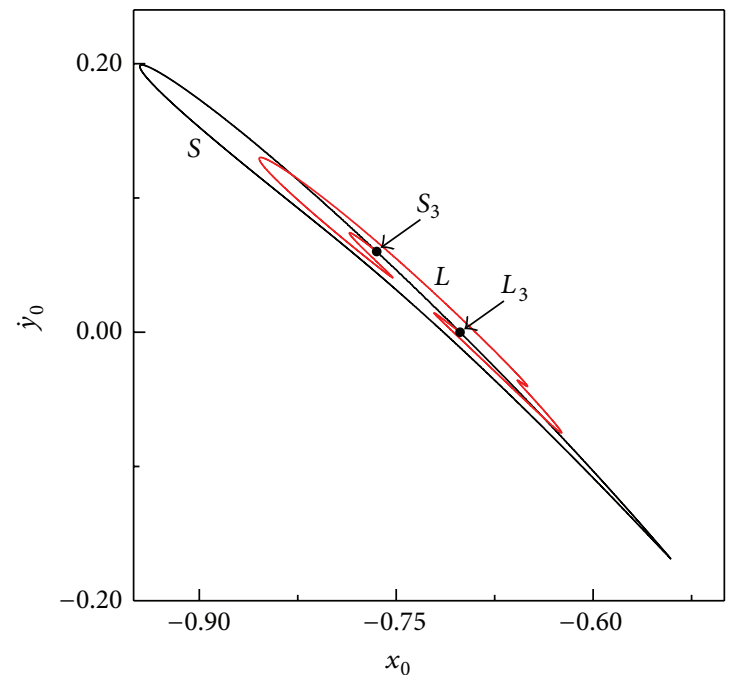

(c)

Figure 13: Projections, in the $\left(x_{0}, \dot{y}_{0}\right)$ plane, of the characteristic curves of long (red color) and short (black color) period families emanating from the collinear equilibria for $\mu=0.2, \omega=0.5$ and radiation factors: (a) $q_{1}=0.015$ and $q_{2}=0.05$ for $L_{1}$, (b) $q_{1}=0.9$ and $q_{2}=0.03$ for $L_{2}$, and (c) $q_{1}=0.03$ and $q_{2}=0.9$ for $L_{3}$.

constant $C$ as well as the horizontal and vertical stability parameters $s_{h}$ and $s_{v}$.

For the short and long period families emanating from the collinear equilibrium points when these are stable we present the corresponding diagrams in Figure 13, as examples of their evolution, for mass parameter $\mu=0.2$ and angular velocity $\omega=0.5$ and for pair values of the radiation factors $\left(q_{1}, q_{2}\right)=(0.015,0.05),(0.9,0.03)$ and $(0.03,0.9)$ for $L_{1}, L_{2}$, and $L_{3}$, respectively. As we see, in Figure 13(a), the long period family emanating from the inner collinear equilibrium point $L_{1}$ terminates at this point while, in Figure 13(b), the long period family emanating from the outer collinear equilibrium point $L_{2}$ to the right of the smaller primary terminates at a bifurcation orbit which belongs to the short period family travelling it three times. The latter is also observed for the long period family emanating from the equilibrium point $L_{3}$ to the left of the larger primary (Figure 13(c)).

With regard to the short period families, at all collinear equilibrium points, they emanate from and terminate at their corresponding equilibria. In particular, the short period family emanating from $L_{1}$ starts with simple periodic orbits around the equilibrium point (see the ellipse in Figure 14(a)) but in its evolution a new vertical intersection with the $\mathrm{O} x$ axis appears (Figures 14(b) and 14(c)) continuing thus with double periodic orbits. Then, this new vertical intersection approaches the initial vertical intersection (Figure $14(\mathrm{~d})$ ) and at approximately the point $\left(x_{0}, \dot{y}_{0}\right)=(0.161656,0.033073)$ these two vertical intersections coincide and then the family returns to the equilibrium point with the double period of the initial one; that is, it travels two times around $L_{1}$. 


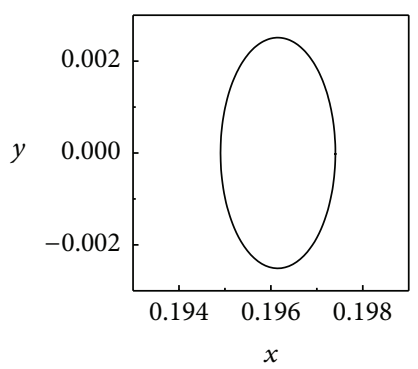

(a)

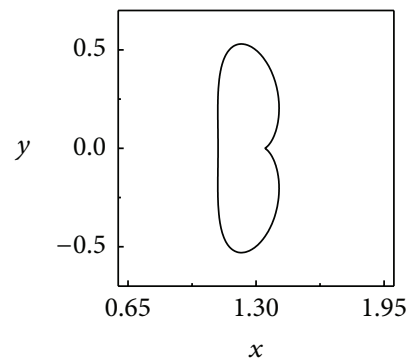

(e)

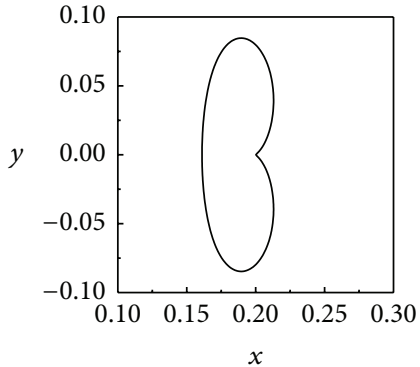

(b)

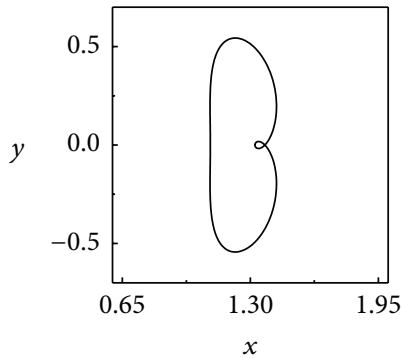

(f)

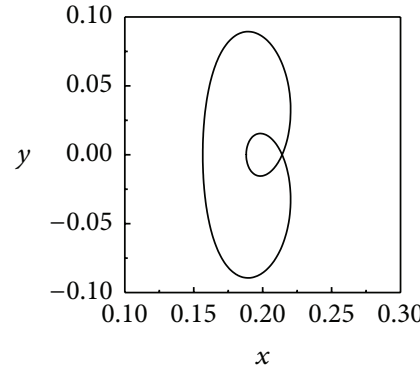

(c)

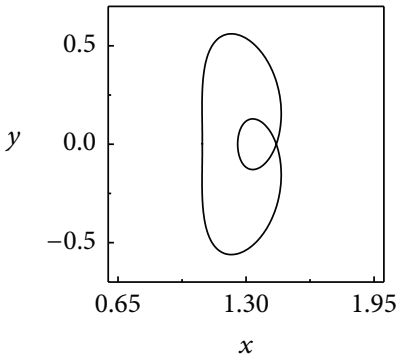

(g)

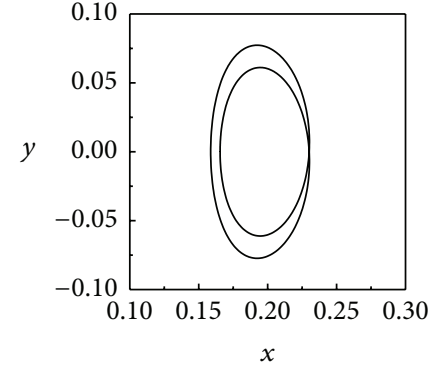

(d)

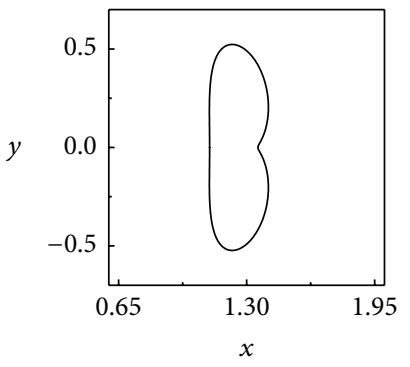

(h)

FIGURE 14: Sample members of the short period families emanating from the stable equilibrium points $L_{1}[(\mathrm{a})-(\mathrm{d})]$ and $L_{2}[(\mathrm{e})-(\mathrm{h})]$.

TABLE 4: Sample members of the short and long period families emanating from the collinear equilibrium points for $\mu=0.2$ and $\omega=0.5$ and the following radiation factors: $q_{1}=0.015$ and $q_{2}=0.05$ for $L_{1}, q_{1}=0.9$ and $q_{2}=0.03$ for $L_{2}$, and $q_{1}=0.03$ and $q_{2}=0.9$ for $L_{3}$.

\begin{tabular}{lccccc}
\hline & $T / 2$ & $x_{0}$ & $\dot{y}_{0}$ & $C$ & $a_{h}$ \\
\hline$S\left(L_{1}\right)$ & 6.721506 & 0.2232037 & -0.0252754 & 0.1032007 & -0.9880 \\
$L\left(L_{1}\right)$ & 14.627908 & 0.1815907 & 0.0111466 & 0.1033552 & 0.7358 \\
$S\left(L_{2}\right)$ & 8.087888 & 1.7300000 & -0.3327057 & 1.3965491 & 4.4041 \\
$L\left(L_{2}\right)$ & 19.595694 & 1.2147080 & 0.1069103 & 1.4042630 & 0.9610 \\
$S\left(L_{3}\right)$ & 13.071411 & -0.7991938 & 0.0721319 & 0.4596958 & -0.1155 \\
$L\left(L_{3}\right)$ & 15.920543 & -0.6517328 & -0.0378064 & 0.4589966 & 0.8108 \\
\hline
\end{tabular}

The same pattern of evolution also appears for the short period family emanating from the collinear equilibrium point $L_{3}$ while for the corresponding family emanating from $L_{2}$ this starts with simple periodic orbits which become double (Figures 14(e)-14(g)). However, in its evolution the new vertical intersection does not approach the other vertical intersection but it disappears (Figure 14(h)); therefore, the family continues again with simple periodic orbits and it returns to the equilibrium point with the same period. Note here that all the corresponding short and long period families consist of periodic orbits which are symmetrical with respect to the $\mathrm{O} x$-axis.

Initial conditions for representative periodic orbits of these families are presented in Table 4, where we give the half period, the abscissa $x_{0}$ on the Ox-axis, the velocity component $\dot{y}_{0}\left(\dot{x}_{0}=0\right.$ at the half period of a symmetric periodic orbit), the value of the Jacobi constant $C$, and the horizontal and vertical stability parameters $a_{h}$ and $a_{v}$.

\section{Conclusions}

Some basic dynamical features of the photogravitational Chermnykh's restricted three-body problem were studied. In this problem, the primaries are considered to be radiating sources with mass reduction factors $q_{1}, q_{2}$, due to the radiation, and the massless body is moving in the orbital plane of the radiating primaries which is rotating with constant angular velocity $\omega$ around the center of mass.

With regard to the planar equilibrium points it was found that, as in the classical case, the problem admits five stationary solutions and, in particular, three collinear and two triangular. An interesting result is that the triangular equilibrium points may fall onto the $\mathrm{O} x$-axis, where they coalesce with the collinear equilibrium points. Specifically, they coalesce with the inner collinear point $L_{1}$ when $q_{1}^{1 / 3}+$ $q_{2}^{1 / 3}=\omega^{2 / 3}$ and with the outer collinear points $L_{2}, L_{3}$ when $\left|q_{1}^{1 / 3}-q_{2}^{1 / 3}\right|=\omega^{2 / 3}$. When these equations hold, the triangular equilibrium points transfer their stability to the corresponding collinear points. For all equilibria, stability criteria with respect to the parameters of the problem were established and several stability regions were plotted. An interesting result is that, in the special case of two primaries with equal mass reduction factors (due to the radiation), linear stability of the triangular equilibria occurs at least for values of the mass parameter which are less than the unique 
value $\mu_{0}=(3-2 \sqrt{2}) / 6$, independently of the values of the other parameters of the problem. Comparing these results to previous results on the photogravitational restricted threebody problem and the corresponding Chermnykh's problem we note that, for both of those problems, the triangular equilibrium points have been found to coalesce only with the inner collinear equilibrium point transferring to it their stability while for the classical case it is known that for each value of the mass parameter the triangular points are fixed at $x=1 / 2-\mu$ and $y= \pm \sqrt{3} / 2$.

For all cases of stable collinear equilibrium points, the motion around them is bounded and is composed of two harmonic motions, giving rise to short and long period families, which consist of symmetric periodic orbits. Note that, for the corresponding Chermnykh's or photogravitational problems and with respect to the specific equilibria, these families may be found only for the inner collinear equilibrium point, while for the classical problem, it is known that only the unstable Lyapunov families emanate from them. Certain examples of the short and long period families were given to show their existence and evolution. For the considered short period families of our examples we found that each one of them terminates at its corresponding equilibrium point.

Concerning the respective families emanating from the triangular equilibrium points, specific examples were drawn showing that their characteristic curves have a similar evolution with that of the corresponding families of the classical problem, which was also examined thoroughly. Specifically, the short period family terminates on the Lyapunov family emanating from the outer collinear equilibrium point to the left of the larger primary, while the long period family forms bridges connecting orbits of the short period family which are bifurcation points among these two families. The corresponding families consist of asymmetric periodic orbits. To the authors' knowledge, related results for the Chermnykh or the photogravitational restricted three-body problem showing explicitly the evolution of the long period family do not exist in the literature.

A natural extension of the present study is to consider negative values for the radiation factors, a case for which at least new equilibrium points out of the orbital plane are allowed to exist. Another interesting case for study would be also to incorporate the Poynting-Robertson relativistic correction in the radiation force as well as to consider the belt gravitational potential as an additional term in the potential function.

\section{Conflict of Interests}

The authors declare that there is no conflict of interests regarding the publication of this paper.

\section{Acknowledgment}

A. A. Nikaki acknowledges financial support under University of Patras "K. Karatheodory" research grant.

\section{References}

[1] V. G. Szebehely, Theory of Orbits, the Restricted Problem of Three Bodies, Academic Press, 1967.

[2] J. E. Marsden and S. D. Ross, "New methods in celestial mechanics and mission design," Bulletin of the American Mathematical Society, vol. 43, pp. 43-73, 2005.

[3] R. Prosmiti, S. C. Farantos, R. Guantes, F. Borondo, and R. M. Benito, "A periodic orbit analysis of the vibrationally highly excited LiNC/LiCN: a comparison with quantum mechanics," The Journal of Chemical Physics, vol. 104, no. 8, pp. 2921-2931, 1996.

[4] M. N. Vrahatis, A. E. Perdiou, V. S. Kalantonis et al., "Application of the characteristic bisection method for locating and computing periodic orbits in molecular systems," Computer Physics Communications, vol. 138, no. 1, pp. 53-68, 2001.

[5] M. M. Sano, "Dynamics starting from zero velocities in the classical Coulomb three-body problem," Physical Review E, vol. 75, no. 2, Article ID 026203, 2007.

[6] S. V. Chermnykh, "Stability of libration points in a gravitational field," Leningradskii Universitet Vestnik Matematika Mekhanika Astronomiia, vol. 2, pp. 73-77, 1987.

[7] K. Goździewski and A. J. Maciejewski, "Nonlinear stability of the Lagrangian libration points in the Chermnykh problem," Celestial Mechanics \& Dynamical Astronomy, vol. 70, no. 1, pp. 41-58, 1998.

[8] E. A. Perdios and O. Ragos, "Asymptotic and periodic motion around collinear equilibria in Chermnykh's problem," Astronomy and Astrophysics, vol. 414, no. 1, pp. 361-371, 2004.

[9] K. E. Papadakis, "The 3D restricted three-body problem under angular velocity variation," Astronomy \& Astrophysics, vol. 425, no. 3, pp. 1133-1142, 2004.

[10] A. E. Perdiou, A. A. Nikaki, and E. A. Perdios, "Periodic motions in the spatial Chermnykh restricted three-body problem," Astrophysics and Space Science, vol. 345, no. 1, pp. 57-66, 2013.

[11] I.-G. Jiang and L.-C. Yeh, "On the Chermnykh-like problems: I. The mass parameter $\mu=0.5$," Astrophysics and Space Science, vol. 305, no. 4, pp. 341-348, 2006.

[12] E. I. Abouelmagd, M. E. Awad, E. M. A. Elzayat, and I. A. Abbas, "Reduction the secular solution to periodic solution in the generalized restricted three-body problem," Astrophysics and Space Science, vol. 350, no. 2, pp. 495-505, 2014.

[13] J. Singh and O. Leke, "Periodic orbits in the Chermnykh-like restricted problem of oblate bodies with radiation," Astrophysics and Space Science, vol. 350, no. 1, pp. 109-117, 2014.

[14] J. Singh and O. Leke, "Motion in a modified Chermnykh's restricted three-body problem with oblateness," Astrophysics and Space Science, vol. 350, no. 1, pp. 143-154, 2014.

[15] V. V. Radzievskii, "The restricted three-body problem including radiation pressure," Astronomicheskii Zhurnal, vol. 27, pp. 250256, 1950.

[16] T. J. Kalvouridis, "On some new aspects of the photogravitational Copenhagen problem," Astrophysics and Space Science, vol. 317, no. 1-2, pp. 107-117, 2008.

[17] R. Bewick, J. Sanchez, and C. R. McInnes, "The feasibility of using an $\mathrm{L}_{1}$ positioned dust cloud as a method of space-based geoengineering," Advances in Space Research, vol. 49, no. 7, pp. 1212-1228, 2012.

[18] J. F. Simmons, A. J. McDonald, and J. C. Brown, “The restricted 3-body problem with radiation pressure," Celestial Mechanics, vol. 35, no. 2, pp. 145-187, 1985. 
[19] K. Goździewski, A. J. Maciejewski, and Z. Niedzielska, "About stability of libration points in the restricted photogravitational three body problem," Celestial Mechanics and Dynamical Astronomy, vol. 52, no. 2, pp. 195-201, 1991.

[20] A. Elipe and M. Lara, "Periodic orbits in the restricted three body problem with radiation pressure," Celestial Mechanics and Dynamical Astronomy, vol. 68, no. 1, pp. 1-11, 1997.

[21] E. A. Perdios, S. S. Kanavos, and V. V. Markellos, "Bifurcations of plane to 3D periodic orbits in the photogravitational restricted three-body problem," Astrophysics and Space Science, vol. 278, no. 4, pp. 407-413, 2001.

[22] E. A. Perdios, "Critical symmetric periodic orbits in the photogravitational restricted three-body problem," Astrophysics and Space Science, vol. 286, no. 3-4, pp. 501-513, 2003.

[23] A. S. Zimovshikov and V. N. Tkhai, "Stability diagrams for a heterogeneous ensemble of particles at the collinear libration points of the photogravitational three-body problem," Journal of Applied Mathematics and Mechanics, vol. 74, no. 2, pp. 158$163,2010$.

[24] E. I. Abouelmagd, "The effect of photogravitational force and oblateness in the perturbed restricted three-body problem," Astrophysics and Space Science, vol. 346, no. 1, pp. 51-69, 2013.

[25] J. Singh and J. J. Taura, "Stability of triangular equilibrium points in the photogravitational restricted three-body problem with oblateness and potential from a belt," Journal of Astrophysics and Astronomy, vol. 35, no. 2, pp. 107-119, 2014.

[26] D. G. Yárnoz, J. P. S. Cuartielles, and C. R. McInnes, "Passive sorting of asteroid material using solar radiation pressure," Journal of Guidance, Control, and Dynamics, vol. 37, pp. 12231235, 2014.

[27] P. Verrier, T. Waters, and J. Sieber, "Evolution of the $\mathrm{L}_{1}$ halo family in the radial solar sail circular restricted three-body problem," Celestial Mechanics and Dynamical Astronomy, vol. 120, no. 4, pp. 373-400, 2014.

[28] J. Henrard, “On Brown's conjecture," Celestial Mechanics, vol. 31, no. 2, pp. 115-122, 1983.

[29] Y. A. Chernikov, "The photogravitational restricted three-body problem," Soviet Astronomy, vol. 14, pp. 176-181, 1970.

[30] A. L. Kunitsyn and A. T. Tureshbaev, "On the collinear libration points in the photo-gravitational three-body problem," Celestial Mechanics, vol. 35, no. 2, pp. 105-112, 1985.

[31] M. K. Das, P. Narang, S. Mahajan, and M. Yuasa, "Effect of radiation on the stability of equilibrium points in the binary stellar systems: RW-Monocerotis, Krüger 60," Astrophysics and Space Science, vol. 314, no. 4, pp. 261-274, 2008.

[32] D. W. Schuerman, "The restricted three-body problem including radiation pressure," The Astrophysical Journal, vol. 238, no. 1, pp. 337-342, 1980.

[33] J. Henrard, "The web of periodic orbits at $L_{4}$," Celestial Mechanics and Dynamical Astronomy, vol. 83, no. 1-4, pp. 291-302, 2002.

[34] E. A. Perdios and C. G. Zagouras, "Vertical stability of periodic solutions around the triangular equilibrium points," Celestial Mechanics and Dynamical Astronomy, vol. 51, no. 1, pp. 75-81, 1991.

[35] X. Y. Hou and L. Liu, "Vertical bifurcation families from the long and short period families around the equilateral equilibrium points," Celestial Mechanics and Dynamical Astronomy, vol. 101, no. 3, pp. 309-320, 2008.

[36] E. F. Goodrich, "Numerical determination of short-period Trojan orbits in the restricted three-body problem," The Astronomical Journal, vol. 71, pp. 88-93, 1966.
[37] M. Hénon, "Exploration numérique du problème restreint II-Masses égales, stabilité des orbites périodiques," Annales d'Astrophysique, vol. 28, pp. 992-1007, 1965.

[38] M. Hénon, "Vertical stability of periodic orbits in the restricted problem I. Equal masses," Astronomy and Astrophysics, vol. 28, pp. 415-426, 1973.

[39] V. V. Markellos, "On the stability parameters of periodic solutions," Astrophysics and Space Science, vol. 43, no. 2, pp. 449458, 1976.

[40] K. E. Papadakis, "Motion around the triangular equilibrium points of the restricted three-body problem under angular velocity variation," Astrophysics and Space Science, vol. 299, no. 2, pp. 129-148, 2005.

[41] A. Deprit, H. Jacques, J. Palmore, J. F. Price, and D. H. Sadler, "The Trojan manifold in the system earth-moon," Monthly Notices of the Royal Astronomical Society, vol. 137, no. 3, pp. 311335, 1967. 

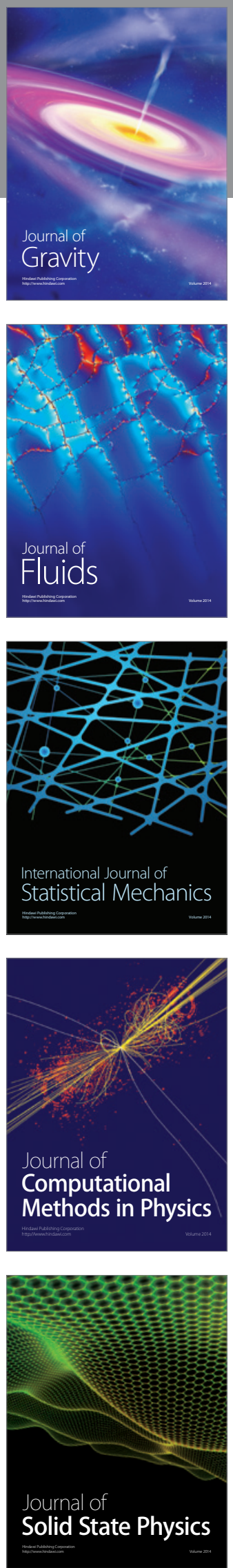

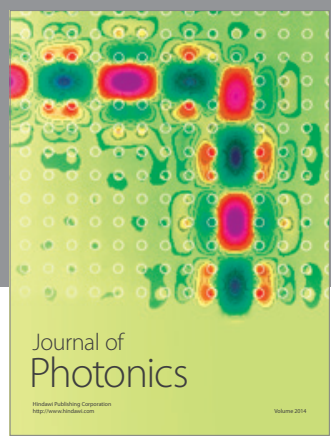

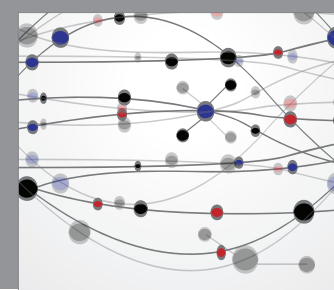

The Scientific World Journal

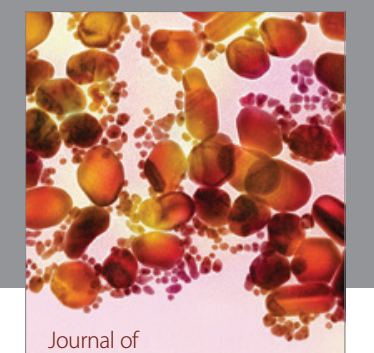

Soft Matter
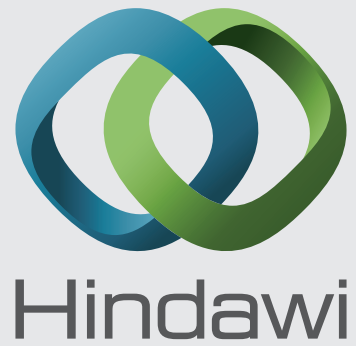

Submit your manuscripts at

http://www.hindawi.com
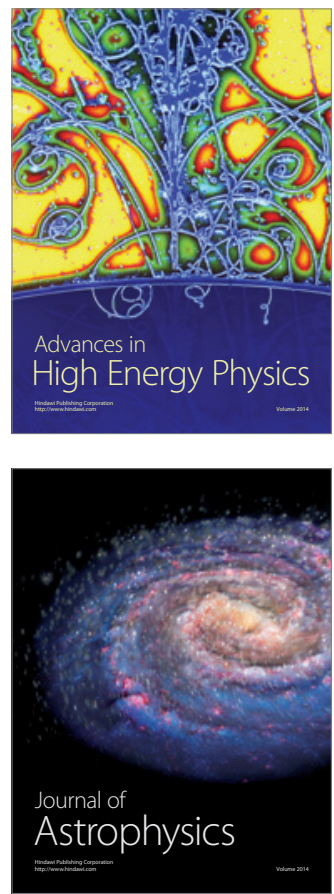
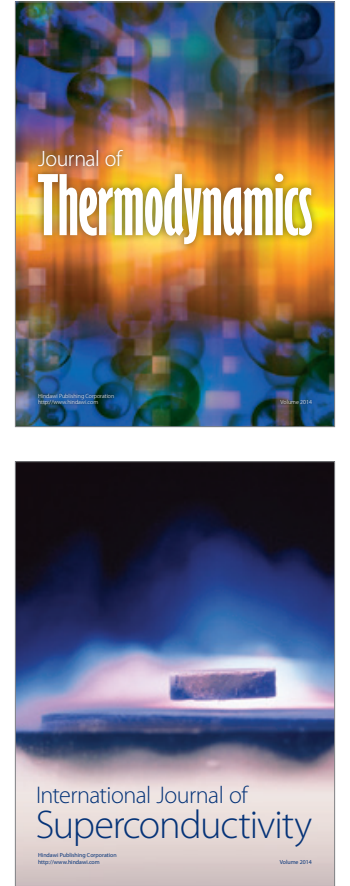
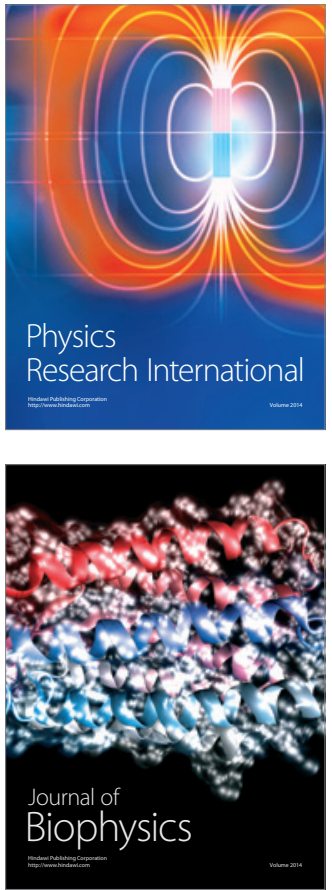
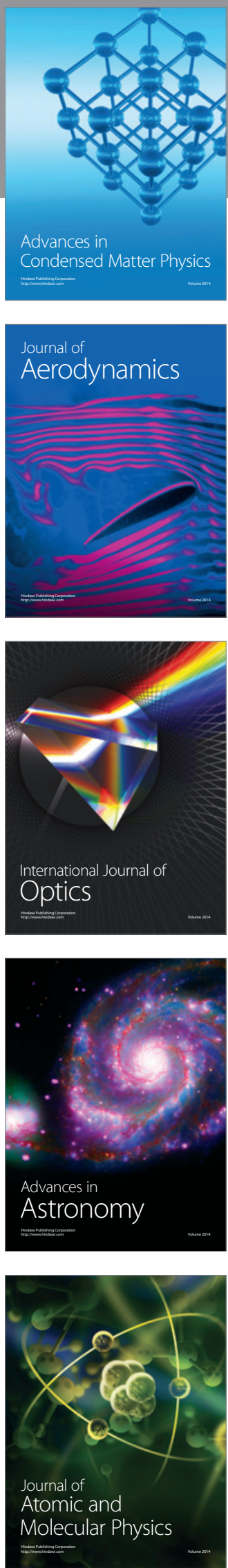